This is a work of the United States Government. In accordance with 17 U.S.C. 105, no copyright protection is available for such works under U.S. Law. Access to this work was provided by the University of Maryland, Baltimore County (UMBC) ScholarWorks@UMBC digital repository on the Maryland Shared Open Access (MD-SOAR) platform.

Please provide feedback

Please support the ScholarWorks@UMBC repository by emailing scholarworks-group@umbc.edu and telling us what having access to this work means to you and why it's important to you. Thank you. 


\section{REPORT DOCUMENTATION PAGE}

Form Approved

OMB NO. 0704-0188

Public Reporting burden for this collection of information is estimated to average I hour per response, including the time for reviewing instructions, searching existing data sources,

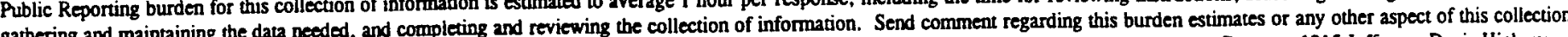

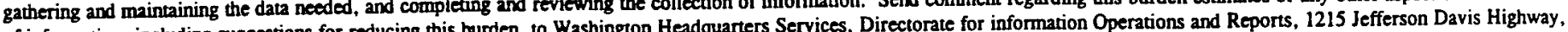

of information, including suggestions for reducing this burden, to Washington Headquarters Services, Directorate for informaction Project (0704-0188,) Washington, DC 20503.
Suite 1204, Arlington, VA 22202-4302, and to the Office of Management and Budget, Paperwork Reduction

\begin{tabular}{|l|l|l|}
\hline 1. AGENCY USE ONLY ( Leave Blank) & 2. REPORT DATE & $\begin{array}{l}\text { 3. REPORT TYPE AND DATES COVERED } \\
\text { Final }\end{array}$ \\
\hline $\begin{array}{l}\text { 4. TIAUg98-31Jul99 } \\
\text { Multi-Photon Entanglement and Quantum Teleportation }\end{array}$ & $\begin{array}{l}\text { 5. FUNDING NUMBERS } \\
\text { DAAG55-98-1-0372 }\end{array}$ \\
$\begin{array}{l}\text { 6. AUTHOR(S) } \\
\text { Yanhua Shih }\end{array}$ & \\
\hline $\begin{array}{l}\text { 7. PERFORMING ORGANIZATION NAME(S) AND ADDRESS(ES) } \\
\text { University of Maryland, Baltimore County }\end{array}$ & $\begin{array}{l}\text { 8. PERFORMING ORGANIZATION } \\
\text { REPORT NUMBER }\end{array}$ \\
$\begin{array}{l}\text { 9. SPONSORING / MONITORING AGENCY NAME(S) AND ADDRESS(ES) } \\
\text { U. S. Army Research Office }\end{array}$ & $\begin{array}{l}\text { 10. SPONSORING / MONITORING } \\
\text { AGENCY REPORT NUMBER }\end{array}$ \\
Research Triangle Park, NC 27709-2211 & ARO 38804.16-PH-QC \\
\hline
\end{tabular}

11. SUPPLEMENTARY NOTES

The views, opinions and/or findings contained in this report are those of the author(s) and should not be construed as an official Deparment of the Army position, policy or decision, unless so designated by other documentation.

12 a. DISTRIBUTION / AVAILABILITY STATEMENT

Approved for public release; distribution unlimited.
12 b. DISTRIBUTION CODE

13. ABSTRACT (Maximum 200 words)

The project "Multi-Photon Entanglement and Quantum Teleportation" concerns a series of experimental and theoretical investigations on multi-photon entangled states and the applications, for example, quantum teleportation. The objective of this research is to probe the foundations of quantum theory of entanglement and its fundamental roles in quantum computing and guantum information processing. This research will definitely benefit many scientific and engineering discipline applications.

This report is for the first year investigation of a long term basic research program. The efforts of the first year research are very important for the achievement of our long term goal. Based on the successful results of the first year research, a novel teleportation experiment which has the ability to provide a true " $100 \%$ teleportation" shall be conducted in the second year.

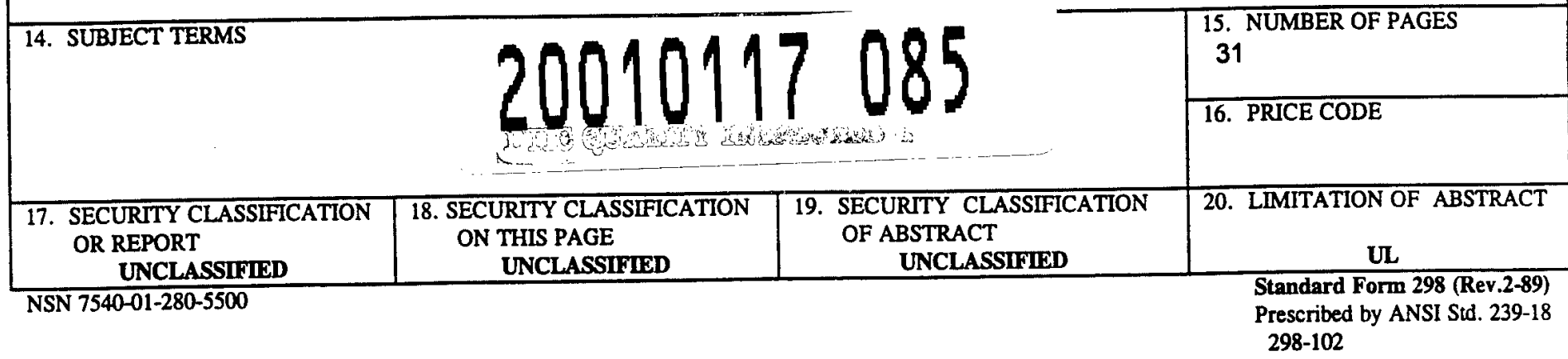




\title{
Multi-Photon Entanglement and Quantum Teleportation
}

(Contract No. DAAG 55-98-1-0372)

FINAL Technical Report Submit to

Army Research Office

ATTN: Dr. Henry Everitt

\author{
PI: Yanhua Shih \\ Department of Physics \\ Lniversity of Maryland. Baltimore County \\ Baltimore. .ID 21250 \\ Telephone: 410-455-2558 (Office). 410-455-2796 (Lab.). \\ 410-455-1072 (FAX). \\ E-mail: Shihâumbc.edu
}

\section{DISTRIBUTION STATEMENT A \\ Approved for Public Release \\ Distribution Unlimited}




\section{Contents}

1 Research Summary 3

2 Accomplishments 3

2.1 Popper's Experiment . . . . . . . . . . . . . . . . . . . . . . . 3

2.2 A Delaved Choice Quantum Eraser . . . . . . . . . . . . . . . . 9

2.3 Two-photon Entangled States and Biphoton Wavefunction . . . . . . . 13

2.4 State Preparation and Quantum Teleportation . . . . . . . . . . . 17

3 On Going Research $\quad 17$

3.1 Three-Photon Entangled States . . . . . . . . . . . . . . . . . 17

3.2 Quantum Teleportation with Complete Set Bell State Measurement . . 24

4 Publications $\quad 27$

5 References 


\section{Research Summary}

The project "Multi-Photon Entanglement and Quantum Teleportation" concerns a series of experimental and theoretical investigations on multi-photon entangled states and the applications. for example. quantum teleportation. The objective of this research is to probe the foundations of quantum theory of entanglement and its fundamental roles in quantum computing and quantum information processing. This research will definitely benefit many scientific and engineering discipline applications.

This report is for the first rear investigation of a long term basic research program. The efforts of the first year research are very important for the achievement of our long term goal. Based on the successful results of the first year research, a novel teleportation experiment which has the ability to provide a true " $100 \%$ teleportation" shall be conducted in the second year.

\section{Accomplishments}

We have demonstrated two interesting experiments recently at UMBC: the historical Popper's experiment [1] and a delayed quantum eraser [2]. The experiments have been reported in several national and international professional conferences and workshops and astonished most of the conference participants. The experimental results started receiving significant attention from the physics community: The Delayed Choice Quantum Eraser experiment has been selected for publication by Physical Review Letters as the first paper of the first issue of the new millennium. We believe these results are very important for the study of foundations of quantum theory, especially, quantum measurement theory. Quantum measurement theory is definitely critical important for quantum computing and quantum information processing. Besides, the Popper's experiment has principally demonstrated a novel technique for N-photon lithography: which has the potential to "beat" the single-photon uncertainty by a factor of $\mathrm{N}$.

\subsection{Popper's Experiment}

The Uncertainty Principle. one of the basic principles of quantum mechanics, distinguishes the world of quantum phenomena from the realm of classical physics. Quantum

mechanics predicts that an exact measurement of both the position and momentum of a particle at the same time is prohibited. We say that the quantum observables "position" and "momentum" are "complementary" because the precise knowledge of the position (momentum) implies that all possible outcomes of measuring the momentum (position) are equally probable.

Karl Popper. being a "metaphysical realist". howerer took a different point of view. In Popper's opinion, the quantum formalism could and should be interpreted realistically: a particle must have precise position and momentum. In this regard he invented a thought experiment in the early 1930's which aimed to support the realistic 
interpretation of quantum mechanics and undermine Copenhagen [4]. What Popper intended to do in his experiment was to show that a particle can have both precise position and momentum at the same time. This is similar to the aim of the EPR gedankenexperiment of 1935 [3]. Unlike EPR's gedankenexperiment, Popper's proposed experiment has been simply ignored by the phrsics community.

We have demonstrated Popper's thought experiment recently. It is indeed astonishing to see that the experimental results agree with Popper's prediction. Through quantum entanglement one may learn the precise knowledge of a photon's position and would therefore expect a greater uncertainty in its momentum. However, the measurement shows that the momentum of this photon does not experience a corresponding increase of uncertainty: Is this a violation of the uncertainty principle?

(a)

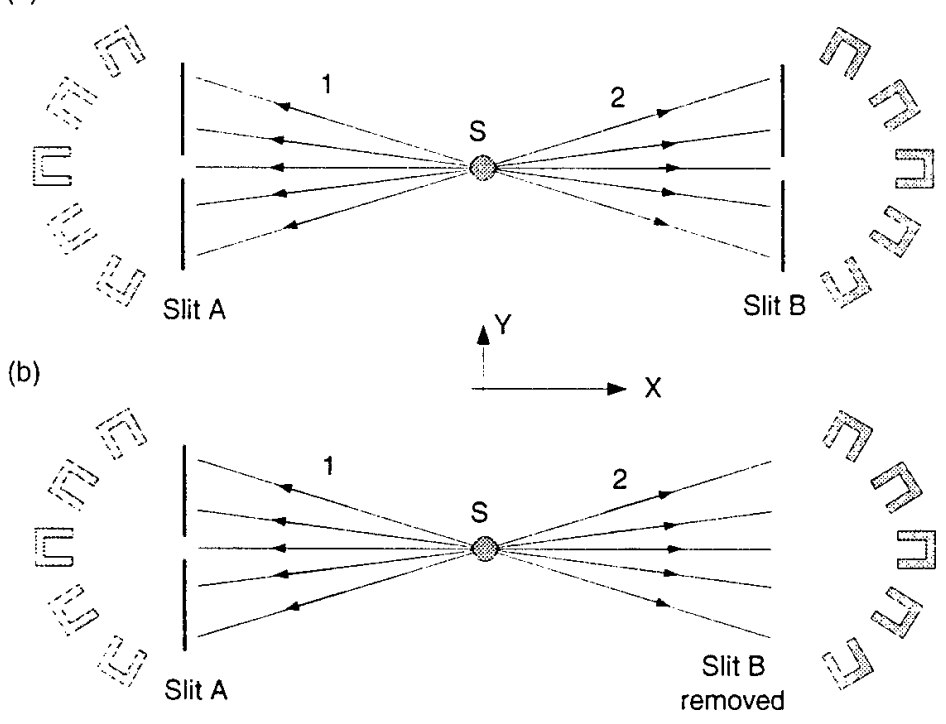

Figure 1: Historical Popper's experiment.

Like EPR's gedankenexperiment. Popper's experiment is based on two-particle entanglement. The entangled EPR type state is a state such that, if the position or momentum of particle 1 is known. the corresponding observable of its twin, particle 2 , is then $100 \%$ determined [3]. There is no quantum rule that forbids this. Popper's original thought experiment is schematically shown in Fig. 1. A point source S, positronium for example. is placed at the center of the experimental arrangement from which entangled pairs of particles 1 and 2 are emitted in opposite directions along the positive and negative $x$-axis towards two screens $A$ and $B$ respectively. There are slits on both screens, parallel to the $y$-axis, and the slits may be adjusted by varying their widths $\Delta y$. Beyond the slits on each side an array of Geiger counters is arranged to detect each of the particles in a pair as shown in the figure. The entangled pair may be emitted to any direction in $4 \pi$ solid angles from the point source. However, if particle 
1 is emitted in a certain direction. particle 2 is known to be moring in the opposite direction, because of momentum conservation for the pair $[5$.

First let us imagine the case in which slits $A$ and $B$ are narrow so that diffraction effects are large. Then all the counters should come into plar: The firing of the counters away from the center line of the slit is indicative of the larger scattering angles, i.e.. the large $\Delta p_{y}$, for each particle due to the narrow slits, i.e.. small $\Delta y$. There seems to be no disagreement in this situation between Copenhagen and Popper. and both sides can provide a reasonable explanation according to their philosophy:

Next imagine that we keep the slit at A very narrow and leave the slit at $B$ wide open. The use of the narrow slit $A$ provides knowledge of the position $y$ of particle 1 and, as a consequence. determines the position of its twin (particle 2 ) on side $B$ due to entanglement. Does particle 2 experience a greater uncertainty in $\Delta p_{y}$ due to the precise knowledge of its position? If not, there comes a serious problem: the product of $\Delta y$ and $\Delta p_{y}$ of particle 2 could be smaller than $h\left(\Delta y \Delta p_{y}<h\right)$. To aviod this problem. it seems that particle 2 going to the left must scatter like its twin which has passed though slit $A$. even though slit $B$ is wide open. Howerer. based on his "statistical-scatter" theory Popper provides a different prediction: particle 2 must not experience a greater $\lambda p_{y}$ unless a real physical narrow slit $B$ is applied.
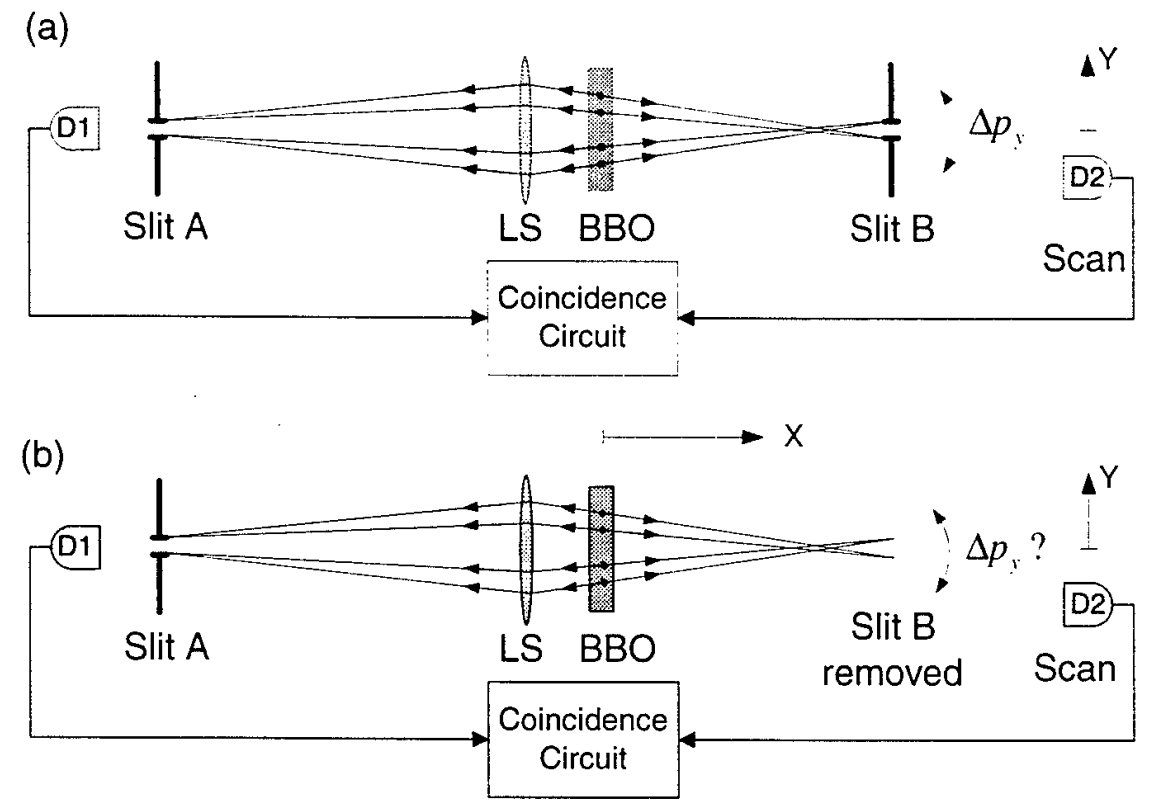

Figure 2: Modified rersion of Popper's experiment.

We have realized Popper's experiment with the use of the entangled two-photon source of spontaneous parametric down conversion (SPDC) [6] [7]. In order to clearly demonstrate all aspects of the historical and modern concerns in a practical manner, Popper's original design is slightly modified as in Fig. 2. The two-photon source is a 
CII Argon ion laser pumped SPDC which provides a two-photon entangled state preserving momentum conservation for the signal-idler photon pair in the SPDC process. By taking advantage of the entanglement nature of the signal-idler pair (also labeled "photon 1" and "photon 2") one could make a "ghost image" of slit A at "screen" B. see Fig. 3. The physical principle of the two-photon "ghost image" has been demonstrated in Ref. 81 .

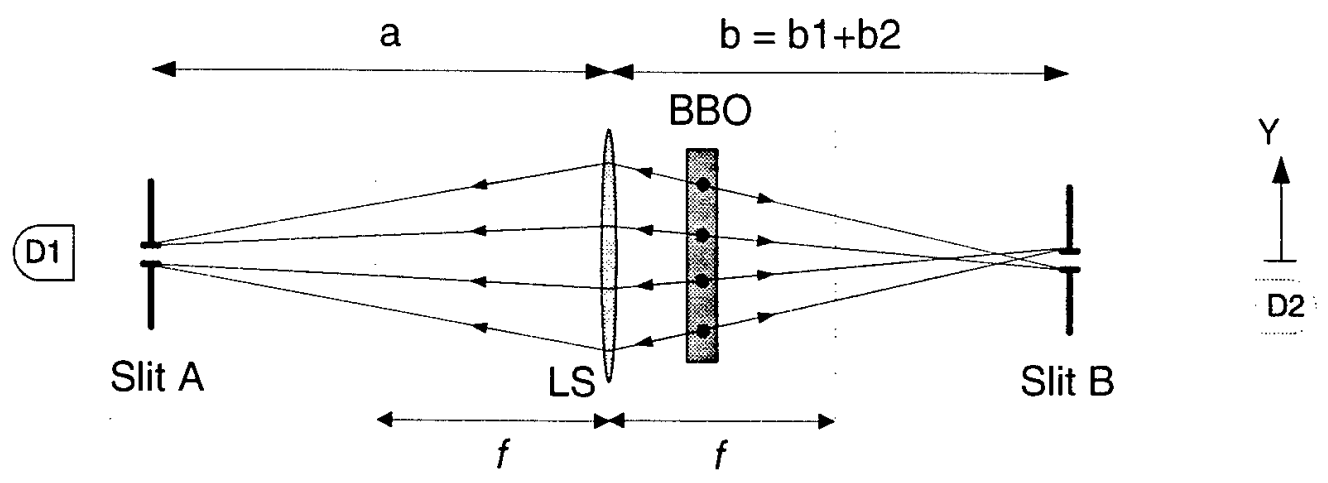

Figure 3: The unfolded schematic of the experiment. It is equivalent to assume $\mathbf{k}_{s}-\mathbf{k}_{i}=0$ but without losing the important entanglement feature of the momentum conservation of the signal-idler pair. It is clear that the locations of slit A, lens LS, and the "ghost image" must be governed by the Gaussian thin lens equations, but bear in mind the different propagation directions of the signal-idler by the small arrows on the straight-line two-photon amplitudes.

The experimental condition required in Popper's experiment is then achiered: when slit $A$ is adjusted to a certain narrow width and slit $B$ is wide open, slit $A$ provides precise knowledge about position of photon 1 on the $y$ axis up to an accuracy $\triangle y$, which equals the width of slit $A$, and the corresponding "ghost image" of pinhole $A$ at "screen" B determines the precise position $y$ of photon 2 to within the same accuracy $\Delta y$. $\Delta p_{y}$ of "photon 2" can be independently studied by measuring the width of its "diffraction pattern" at a certain distance from "screen" B. This is obtained by recording coincidence between detectors $D_{1}$ and $D_{2}$ while scanning detector $D_{2}$ along its $y$ axis. Instead of a battery of Geiger counters, in our experiment only two photon counting detectors $D_{1}$ and $D_{2}$ placed behind the respective silts $A$ and $B$ are used for the coincidence detection. Both $D_{1}$ and $D_{2}$ are driven by step motors and so can be scanned along their $y$ axis. $\Delta y \Delta p_{y}$ of "photon 2 " is then readily calculated and compared with $h$.

The use of a "point source" in the original proposal has been considered as the fundamental mistake Popper made. The basic criticism is that a point source can never produce a pair of entangled particles which preserves two-particle momentum 
conservation. Howerer. a "point source" is not a necessary requirement for Popper's experiment. What we require is the position entanglement of the two-particle system, i.e. if the position of particle 1 is precisely known. the position of particle 2 is also $100 \%$ determined. So that one can learn the precise knowledge of a particle's position through quantum entanglement. Quantum mechanics does allow the position entanglement for an entangled system (EPR state) and there are certain practical mechanisms, such as that the one shown in our experiment, that can be used to realize it.

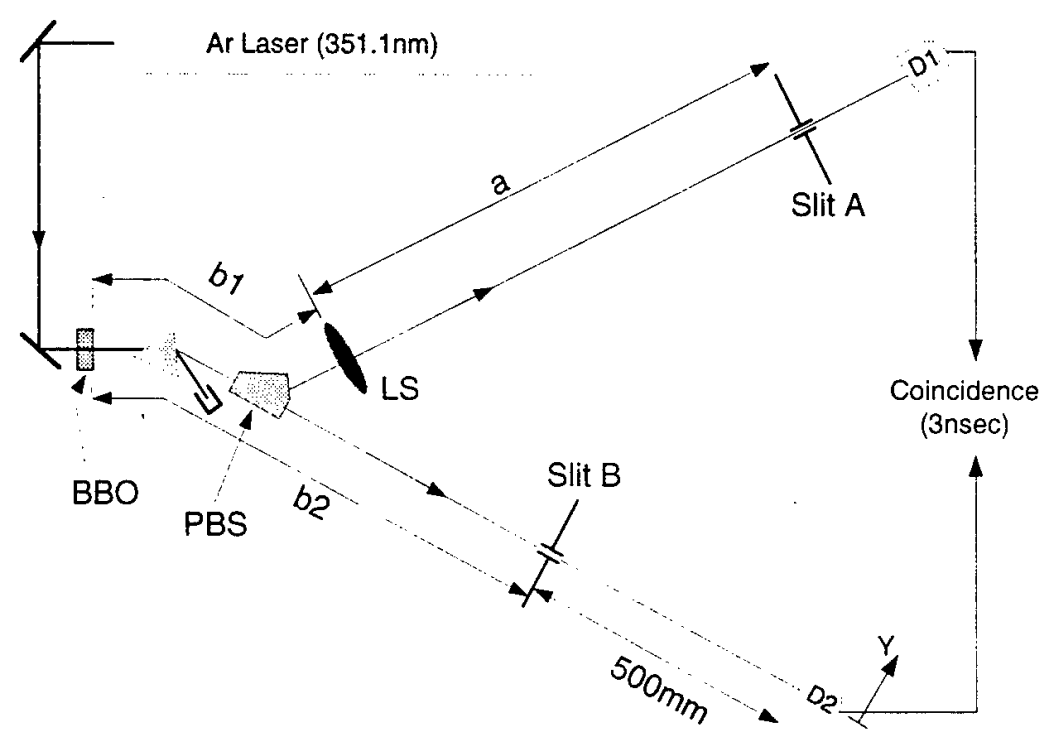

Figure 4: Schematic of the experimental setup.

The schematic experimental setup is shown in Fig. 4 with detailed indications of distances. $A \mathrm{CW}$ Argon ion laser line of $\lambda_{p}=351.1 \mathrm{~nm}$ is used to pump a $3 \mathrm{~mm}$ long beta barium borate (BBO) crystal for type-II SPDC [6] to generate an orthogonally polarized signal-idler photon pair. The laser beam is about $3 \mathrm{~mm}$ in diameter. It is important not to focus the pump beam so that the phase matching condition, $\mathbf{k}_{s}+\mathbf{k}_{i}=\mathbf{k}_{p}$, is well reinforced in the SPDC process [6], where $\mathbf{k}_{j}(j=s, i . p)$ is the wavevectors of the signal (s), idler (i), and pump (p) respectively: The colinear signal-idler beams. with $\lambda_{s}=\lambda_{i}=702.2 n m=2 \lambda_{p}$ are separated from the pump beam by a fused quartz dispersion prism, and then split by a polarization beam splitter PBS. The signal beam ("photon 1") passes a converging lens LS with a $500 \mathrm{~mm}$ focal length. A $0.16 \mathrm{~mm}$ slit is placed at location $A$ which is $1000 \mathrm{~mm}(=2 f)$ behind the lens LS. The use of LS is to achiere a "ghost image" of slit A $(0.16 \mathrm{~mm})$ at "screen" B, which is at the same optical distance $1000 \mathrm{~mm}(=2 f)$ from LS, however in the idler beam (in the path of "photon 2"). The signal and idler beams are then allowed to pass through the respective slits $A$ and $B$ (real slit B or a "ghost image" of slit $A$ ) and to trigger the two photon counting detectors $D_{1}$ and $D_{2}$. The detectors are Geiger mode avalanche photodiodes which are $180 \mu \mathrm{m}$ in diameter. The output pulses from the detectors are sent to a coincidence 
circuit. During the measurement. detector $D_{1}$ is fixed behind slit A. while detector $D_{2}$ is scanned on the $y_{2}$ axis. by a step motor.

Measurement 1: we first studied the case in which both slit $A$ and $B$ were adjusted to be $0.16 \mathrm{~mm}$. The $y$ coordinate of $D_{1}$ was chosen to be 0 (center) while $D_{2}$ was allowed to scan along its $y$ axis. The circled dot data points in Fig. 5 show the coincidence counting rates against the $y$ coordinate of $D_{2}$. It is a typical single-slit diffraction pattern with $\Delta y \Delta p_{y}=h$. Nothing is special in this measurement except we have learned the minimum uncertainty width of $\Delta p_{y}$.

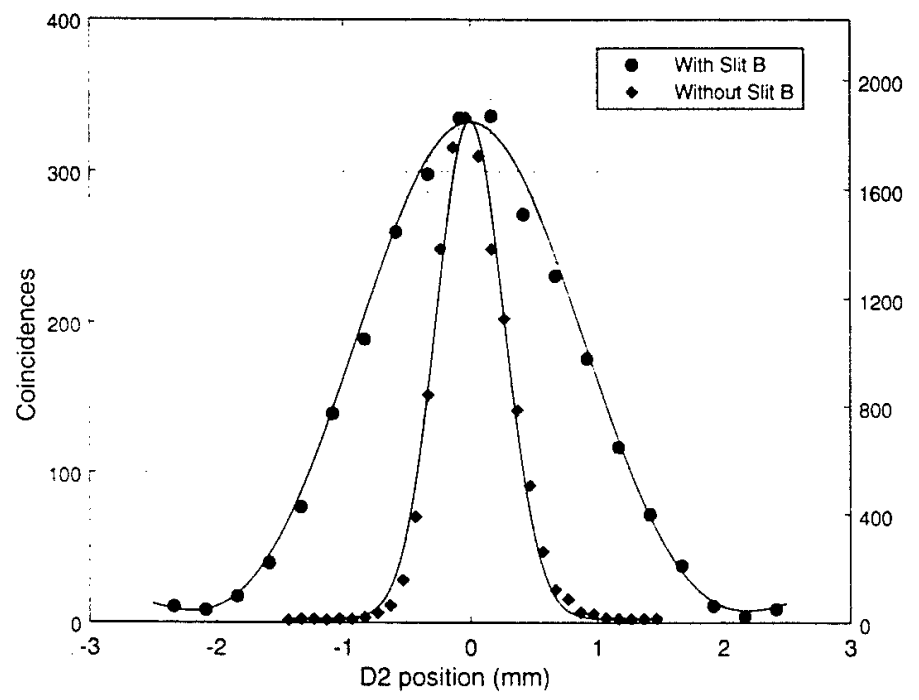

Figure 5: The observed coincidence patterns.

Measurement 2: we kept the same experimental conditions except that slit B was left wide open. This measurement is a test of Popper's prediction. The $y$ coordinate of $D_{1}$ was chosen to be 0 (center) while $D_{2}$ was allowed to scan along its $y$ axis. Due to the entanglement nature of the signal-idler photon pair and the coincidence measurement, only those twins which have passed through slit $A$ and the "ghost image" of slit A at "screen" B with an uncertainty of $\Delta y=0.16 \mathrm{~mm}$. which is the same width as the real slit $B$ we have used in measurement 1. would contribute to the coincidence counts through the triggering of $D_{1}$ and $D_{2}$. The diamond dot data points in Fig. 5 report the measured coincidence counting rates against the $y$ coordinate of $D_{2}$. The measured width of the pattern is narrower than that of the diffraction pattern shown in measurement 1. In addition. the width of the pattern is also much narrower then the actual size of the diverging SPDC beam at $D_{2}$. It is clear that the experimental data indicated $\Delta y \Delta p_{y}<h$ for "photon 2".

Is this a violation of the quantum uncertainty principle? As we shall see, it is not, but illustrates the fact that one should not think of two-particle entangled states as 
composed of single particles in definite states.

Even though we still hare many questions regard to the fundamental issues, the experimental result has shined light on a novel technology of multi-photon lithography. The multi-photon diffraction pattern can have a much narrower width. If designing the N-photon lithography system correctly, one can "beat" the single-photon uncertainty by a factor of $\mathrm{N}$.

\subsection{A Delayed Choice Quantum Eraser}

The idea of "quantum eraser" was proposed by Scully and Drühl in 1982 [9]. Scully has commended that our experiment is the "closest" one of reflecting his original idea. It is truly surprising to see that one could simultaneously observing both particle-like and wave-like behavior of a quantum in one set of measurements. Moreover, the whichpath or both-path information of a quantum can be erased or marked by its entangled twin even after the registration of the quantum.

In 1927, Niels Bohr illustrated complementarity with "ware-like" and "particle-like" attributes of a quantum mechanical object [10]. Since then. complementarity has often been superficially identified with "wave-particle duality of matter". Over the years the two-slit interference experiment has been emphasized as a good example of the enforcement of complementarity: Feynman discussing the two-slit experiment, noted that this wave-particle dual behavior contains the basic mystery of quantum mechanics [11]. The actual mechanisms that enforce complementarity vary from one experimental situation to another. In the two-slit experiment, the common "wisdom" is that the position-momentum uncertainty relation $\delta x \delta p \geq h$ makes it impossible to determine which slit the photon (or electron) passes through without at the same time disturbing the photon (or electron) enough to destroy the interference pattern. However, it has been proven [9] that under certain circumstances this common interpretation may not be true. In 1982. Scully found a way around this position-momentum uncertainty obstacle and proposed a quantum eraser to obtain which-path or particle-like information without scattering or otherwise introducing large uncontrolled phase factors to disturb the interference. To be sure the interference pattern disappears when which path information is obtained. But it reappears when we erase (quantum erasure) the which path information [12,9].

One quantum eraser experiment very close to the 1982 proposal is illustrated in Fig.6. Two atoms labeled by $A$ and $B$ are excited by a laser pulse. $A$ pair of entangled photon. photon 1 and photon 2. is then emitted from either atom $A$ or atom $B$ by atomic cascade decar. Photon 1. propagates to the right. is registered by a photon counting detector $D_{0}$. which can be scanned by a step motor along its $x$-axis for the observation of interference fringes. Photon 2, propagates to the left, is injected into a beamsplitter. If the pair is generated in atom $A$, photon 2 will follow the $A$ path meeting $B S . A$ with $50 \%$ chance of being reflected or transmitted. If the pair is generated in atom $\mathrm{B}$. photon 2 will follow the B path meeting $B S B$ with $50 \%$ chance 


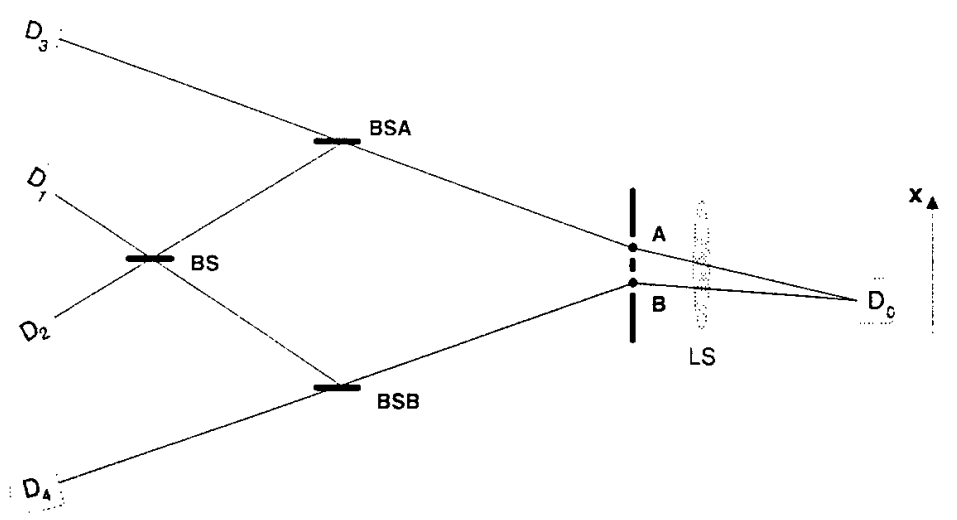

Figure 6: A proposed quantum eraser experiment.

of being reflected or transmitted. Under the $50 \%$ chance of being transmitted by either $B S A$ or $B S B$, photon 2 is detected by either detector $D_{3}$ or $D_{4}$. The registration of $D_{3}$ or $D_{4}$ provides which-path information (path A or path B) of photon 2 and in turn provides which-path information of photon 1 because of the entanglement nature of the two-photon state of atomic cascade decar: Given a reflection at either BS.A or BSB photon 2 will continue to follow its A path or B path to meet another $50-50$ beamsplitter $B S$ and then be detected by either detector $D_{1}$ or $D_{2}$, which are placed at the output ports of the beamsplitter $B S$. The triggering of detectors $D_{1}$ or $D_{2}$ erases the whichpath information. So that either the absence of the interference or the restoration of the interference can be arranged via an appropriately contrived photon correlation study: The experiment is designed in such a way that $L_{0}$, the optical distance between atoms $\mathrm{A}, \mathrm{B}$ and detector $D_{0}$, is much shorter than $L_{i}$, which is the optical distance between atoms $A, B$ and detectors $D_{1} . D_{2} . D_{3}$. and $D_{4}$, respectively. So that $D_{0}$ will be triggered much earlier by photon 1 . After the registration of photon 1 . we look at these "delayed" detection events of $D_{1}, D_{2}, D_{3}$, and $D_{4}$ which have constant time delavs, $\tau_{i} \simeq\left(L_{i}-L_{0}\right) / c$, relative to the triggering time of $D_{0}$. It is easy to see these "joint detection" events must have resulted from the same photon pair. It was predicted that the "joint detection" counting rate $R_{01}$ (joint detection rate between $D_{0}$ and $D_{1}$ ) and $R_{02}$ will show interference pattern when detector $D_{0}$ is scanned along its $x$-axis. This reflects the wave property (both-path) of photon 1 . However, no interference will be observed in the "joint detection" counting rate $R_{03}$ and $R_{04}$ when detector $D_{0}$ is scanned along its $x$-axis. This is clearly expected because we now have indicated the particle property (which-path) of photon 1. It is important to emphasize that all four "joint detection" rates $R_{01}, R_{02}, R_{03}$, and $R_{04}$ are recorded at the same time during one scanning of $D_{0}$ along its $y$-axis. That is. in the present experiment we "see" both wave (interference) and which-path (particle-like) with the same apparatus.

The schematic diagram of our experimental set up is shown in Fig. 7 . Instead of atomic cascade decay. spontaneous parametric down conversion (SPDC) is used to 


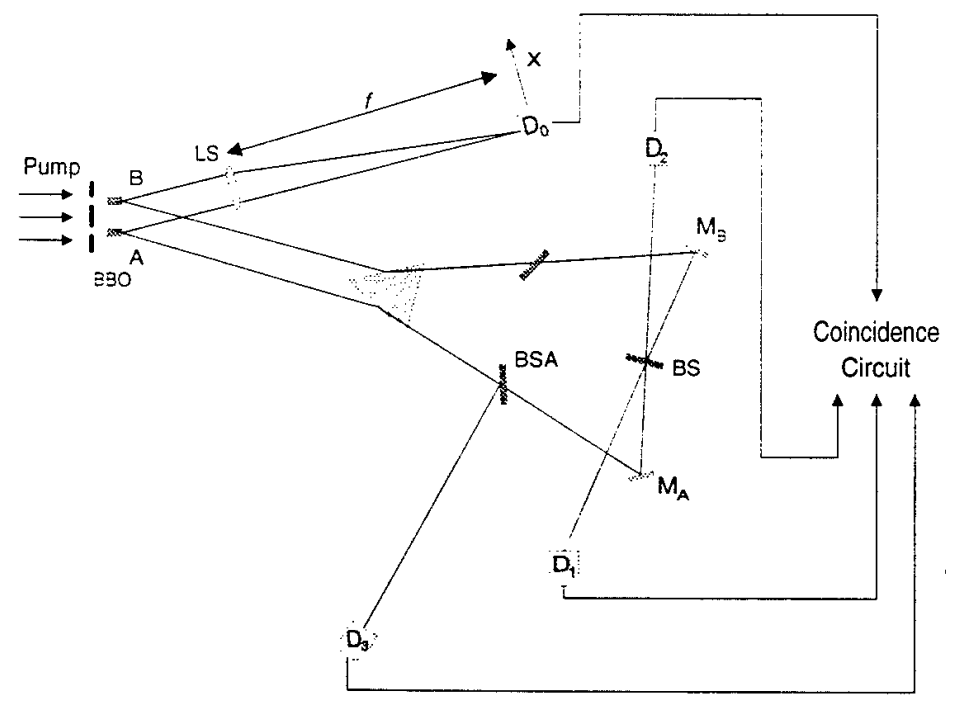

Figure 7: Schematic of the experimental setup.

prepare the entangled two-photon state. In this experiment. the $351.1 \mathrm{~nm}$ Argon ion pump laser beam is divided by a double-slit and incident onto a type-II phase matching [20] nonlinear optical crystal $\mathrm{BBO}\left(\beta-\mathrm{BaB}_{2} \mathrm{O}_{4}\right)$ at two regions $\mathrm{A}$ and $\mathrm{B}$. A pair of $702.2 \mathrm{~nm}$ orthogonally polarized signal-idler photon is generated either from $\mathrm{A}$ or $\mathrm{B}$ region. The width of the $\mathrm{SPDC}$ region is about $0.3 \mathrm{~mm}$ and the distance between the center of $A$ and $B$ is about $0.7 \mathrm{~mm}$. A Glen-Thompson prism is used to split the orthogonally polarized signal and idler. The signal photon (photon 1, either from A or B) passes a lens $L S$ to meet detector $D_{0}$, which is placed on the Fourier transform plane (focal plane for collimated light beam) of the lens. The use of lens $L S$ is to achieve the "far field" condition: but still keep a short distance between the slit and the detector $D_{0}$. Detector $D_{0}$ can be scanned along its $x$-axis by a step motor. The idler photon (photon 2) is send to an interferometer with equal-path optical arms. The interferometer includes a prism $P S$, two 50-50 beamsplitters $B S A, B S B$, two reflecting mirrors $M_{A}, M_{B}$, and a 50-50 beamspliter $B S$. Detectors $D_{1}$ and $D_{2}$ are placed at the two output ports of the $B S$, respectively, for erasing the which-path information. The triggering of detector $D_{3}$ and $D_{4}$ provide which-path information of the idler (photon 2) and in turn provide which-path information of the signal (photon 1). The electronic output pulses of detectors $D_{1} . D_{2}, D_{3}$, and $D_{4}$ are sent to coincidence circuits with the output pulse of detector $D_{0}$, respectively, for the counting of "joint detection" rates $R_{01}, R_{02}, R_{03}$, and $R_{04}$. In this experiment the optical delav $\left(L_{i}-L_{0}\right)$ is chosen to be $\simeq 2.5 \mathrm{~m}$. where $L_{0}$ is the optical distance between the output surface of $B B O$ and detector $D_{0}$. and $L_{i}$ is the optical distance between the output surface of the $B B O$ and detectors $D_{1}, D_{2} . D_{3}$, and $D_{4}$, respectively. This means that any information one can learn from photon 2 must be at least $8 \mathrm{~ns}$ later than that one has learned from the registration of photon 1 . Compare to the $1 \mathrm{~ns}$ response time of the detectors, $2.5 \mathrm{~m}$ 
delay is good enough for a "delayed erasure".

Fig.8, 9, and 10 report the experimental results. which are all consistent with prediction. Fig. 8 and 9 show the "joint detection" rates $R_{01}$ and $R_{02}$ against the $x$ coordinates of detector $D_{0}$. It is clear we have observed the standard Young's double-slit interference pattern. However. there is a $\pi$ phase shift between the two interference fringes. The $\pi$ phase shift is due to the reflection of the beamsplitter. Fig.10 reports a typical $R_{03}\left(R_{0 t}\right)$. "joint detection" counting rate between $D_{0}$ and "which-path" $D_{3}$ $\left(D_{4}\right)$, against the $x$ coordinates of detector $D_{0}$. An absence of interference is clearly demonstrated. There is no significant difference between the curves of $R_{03}$ and $R_{04}$ except the small shift of the center.

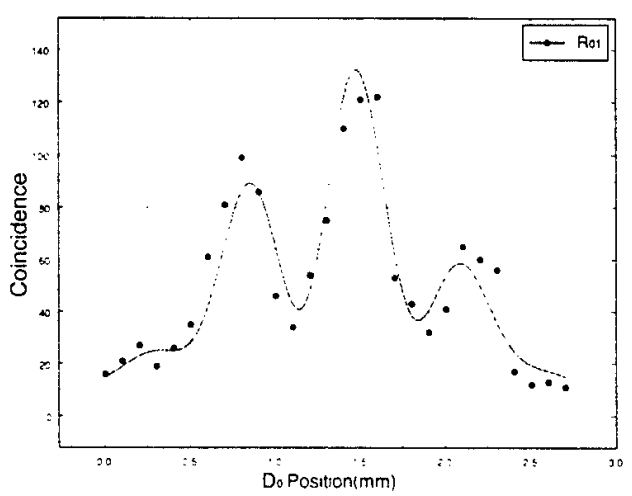

Figure 8: $R_{01}$ ("joint detection" rate between detectors $D_{0}$ and $D_{1}$ ) against the $x$ coordinates of detector $D_{0}$.)

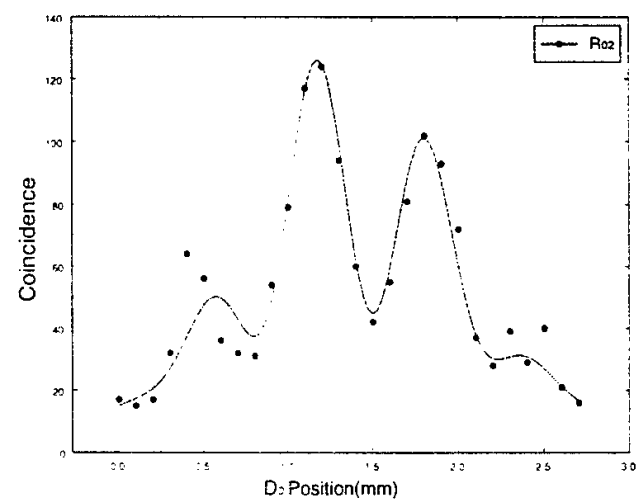

Figure 9: $R_{02}$ ("joint detection" rate between detectors $D_{0}$ and $D_{2}$ ) against the $x$ coordinates of detector $D_{0}$.)

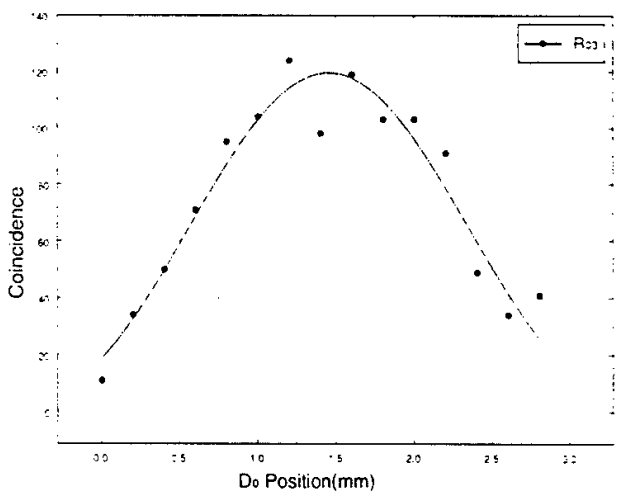

Figure 10: $R_{03}$ ("joint detection" rate between detectors $D_{0}$ and $D_{3}$ ). 
It is true that one could observe both "wave-like" and "particle-like" property of a quantum system in one set of experimental measurements and these information could be learned through its entangled twin event after the registration of the quantum itself. How do we understand this phenomenon?

\subsection{Two-photon Entangled States and Biphoton Wavefunc- tion}

We believe puzzling questions discussed above come from a fundamental misunderstanding of quantum entanglement. Basically, an entangled two-particle system can not be considered as two indiridual particles in definite states. 2 is not $1+1$. As a matter of fact, a maximally entangled two-particle state, such as the EPR state, does not contain any knowledge of the state for either particle 1 or particle 2 individually. Quantum mechanically, an entangled two-particle state only provides the correlation of the pair. Furthermore, the pair is described by one single wavepacket but not by two wavepackets [13]. Two-photon physics is very different from the physics of two classically correlated photons 11].

We strongly believe that it has been a historical mistake to mix up the questions for individual particle behavior with an entangled two-particle system.

The classic example of a two-particle entangled state was suggested by Einstein, Podolsky, and Rosen in their famous 1935 gedankenexperiment [3]:

$$
\Psi\rangle=\sum_{a . b} \delta\left(a+b-c_{0}\right)|a\rangle|b\rangle
$$

where $a, b$ may be the momentum (or position) of particle one and particle two, respectively, and $c_{0}$ is a constant. What is surprising about the entangled state (1) according to EPR is the following: the value of an observable for neither single subsystem is determinate. However. if the measurement of one of the subsystems gives value " $a$ " for that observable, then a measurement of the other subsystem will always give " $c_{0}-a$ " (100\% determination). This "spooky" phenomenon has been experimentally observed $[15]$.

Another example of an entangled two-particle system. suggested br Bohm [16], concerned a singlet state of two spin $1 / 2$ particles:

$$
|\Psi\rangle=\frac{1}{\sqrt{2}}\left(\left|\uparrow_{1}\right\rangle\left|\downarrow_{2}\right\rangle-\left|\downarrow_{1}\right\rangle\left|\uparrow_{2}\right\rangle\right.
$$

where the kets $|\uparrow\rangle$ and $|\downarrow\rangle$ represent states of spin "up" and "down". respectively, along an arbitrary direction. In Bohm's system, using EPR's language the spin of neither particle is determinate. However, if the spin of one particle is measured to be along any direction the other one is $100 \%$ certain to be anti-parallel. This EPR-Bohm type correlation for the polarization of photon pairs has been observed since the early 50 's [17][18]. 
Optical spontaneous parametric down conversion (SPDC) is a typical mechanism to generate EPR type entangled two-photon states. In SPDC. an optical beam. called pump. is incident on a birefringent crystal. The pump is intense enough so that nonlinear interaction lead to the conversion of the pump into a pair of photons. historically called signal and idler. The SPDC is named type I or type II, if the pair has parallel or orthogonal polarizations. respectively. The two-photon state generated from SPDC can be calculated from the first order perturbation theory to be [7],

$$
|\Psi\rangle=\sum_{s, i} \delta\left(\omega_{s}+\omega_{i}-\omega_{p}\right) \delta\left(\mathbf{k}_{s}+\mathbf{k}_{i}-\mathbf{k}_{p}\right) a_{s}^{\dagger}\left(\omega_{s}\left(\mathbf{k}_{s}\right)\right) a_{i}^{\dagger}\left(\omega_{i}\left(\mathbf{k}_{i}\right)\right)|0\rangle
$$

where $i$ and $\mathrm{k}$ represents the frequency and the wave vector for the signal (s), idler (i), and pump (p). The two delta functions in state (3) are usually explicitly written as phase matching conditions:

$$
\omega_{s}+\omega_{i}=\omega_{p}, \quad \mathbf{k}_{s}+\mathbf{k}_{i}=\mathbf{k}_{p}
$$

It is clear that state (3) is an EPR type two-photon entangled state. The state can not be factorized into a product of two single photon states [19]. The physics of entangled two-particle system has been discussed since 1935. It is very important to understand the phrsics behind the mathematics.

The special properties of two-photon physics may be better reflected from its wavefunction. A theory of effective two-photon wavefunction has been dereloped in our group recently [13][20]. As a matter of fact. instead of two wavepackets, it is a wavepacket to describe the entangled two-particle system. The effective two-photon wavefunction $\Psi\left(t_{1}, t_{2}\right)$ will be developed in the following calculation br considering a simple coincidence measurement experiment. in which the signal is sending directly to detector $1 . D_{1}$, while the idler is sending directly to detector $2, D_{2}$. Assume that a type I nonlinear crystal is used for SPDC. so that the signal-idler photon pair have the same polarization. The fields at the detectors 1 and 2 are given by

$$
\begin{aligned}
& E_{1}^{(+)}(t)=\int d \omega f(\omega) e^{-i \omega\left(t-\tau_{1}\right)} a_{s}(\omega) \\
& E_{2}^{(+)}(t)=\int d \omega f(\omega) e^{-i \omega\left(t-\tau_{2}\right)} a_{i}(\omega)
\end{aligned}
$$

where $f(\omega)$ is the spectral distribution function of the field. $a_{j}$ is the annihilation operator of the photons, $j=s, i$, and $\tau_{i} \equiv r_{i} / c$, where $r_{i}$ is the optical path length from the output surface of the nonlinear crystal to the i-th detector, $c$ is the speed of light. We assume $\tau_{1}=\tau_{2}$ to simplify the discussion. The average coincidence counting rate is given by,

$$
\begin{aligned}
R_{c} & =\frac{1}{T} \iint_{0}^{T} d T_{1} d T_{2}\left\langle\Psi\left|E_{1}^{(-)} E_{2}^{(-)} E_{2}^{(+)} E_{1}^{(+)}\right| \Psi\right\rangle \\
& =\frac{1}{T} \iint_{0}^{T} d T_{1} d T_{2}\left|\Psi\left(t_{1}, t_{2}\right)\right|^{2}
\end{aligned}
$$


where $t_{i} \equiv T_{i}-\tau_{i} . T_{i}$ is the detection time of the $\mathrm{i}$-th detector. $T$ is the duration time of the measurement. An effective two-photon warefunction $\Psi\left(t_{1}, t_{2}\right)$ has been defined in $\mathrm{Eq} .(6)$.

$$
\Psi\left(t_{1}, t_{2}\right)=\left\langle 0\left|E_{2}^{(+)} E_{1}^{(-i}\right| \Psi\right\rangle
$$

A simple model of a biphoton generated by pump with a Gaussian spectral function is

$$
\Psi\left(t_{1}, t_{2}\right)=A_{0} e^{-\frac{\sigma_{p}^{2}\left(t_{1}+t_{2}\right)^{2}}{8}} e^{-\frac{\sigma^{2}\left(t_{1}-t_{2}\right)^{2}}{1}} e^{-i \Omega_{s} t_{1}} e^{-i \Omega_{i} t_{2}}
$$

where $\sigma_{p}$ and $\sigma$ are the spectral bandwidths of the pump. the spectral filters for detectors one and two. respectively, $\Omega_{s}, \Omega_{i}$ are the center frequencies of the signal and the idler which are related to the center frequency of the pump, $\Omega_{p}$, by $\Omega_{s}+\Omega_{i}=\Omega_{p}$. Eq. (8) is a two dimensional wavepacket, referred to as the effective two-photon wavefunction or for short the biphoton. Fig.11 is a schematic picture of the two-photon wavepacket. which reflects the entanglement nature of the two-photon state. It is completely different from that of a single photon wavepacket. Also it is very easy to see that this wavefunction can not be considered as a product of two single photon wavefunctions.

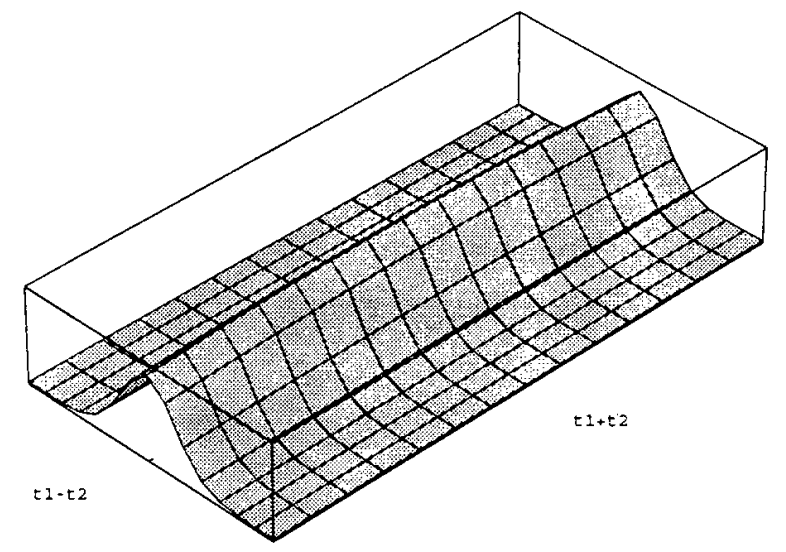

Figure 11: Schematic picture of a biphoton wavepacket.

Trpe II SPDC has a very special nature. which is important for the study of multiphoton entanglement. The two-photon state of type II SPDC is entangled both in space-time (EPR ) and in spin (EPR-Bohm) [21]. In type-II SPDC. the signal-idler pair has orthogonal polarizations. If the signal is measured to be an o-ray of the SPDC crystal the idler must be an $e$-ray and rise versa. A non-collinear type-II SPDC is illustrated in Fig.12. The signal-idler pair are emitted into two cones, one ordinary polarized, the other extraordinary polarized. Along the intersection lines, where the cones orerlap. two pinholes numbered as 1 and 2 are used for defining the direction of 


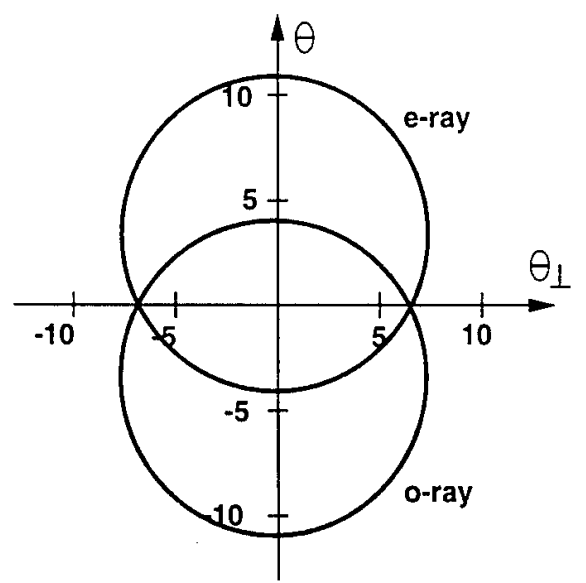

Figure 12: Crossectional view of a degenerate wavelengths non-colinear output cones for a type II SPDC.

the $\mathrm{k}$ vectors of the signal-idler pair. The polarization state of the signal-idler pair can be simplified to the form.

$$
|\Psi\rangle=\frac{1}{\sqrt{2}}\left\{\left|o_{1}\right\rangle\left|e_{2}\right\rangle \pm\left|e_{1}\right\rangle \mid o_{2} \eta_{i}\right.
$$

but keep in mind that the complete state of type II SPDC is in the form of Eq. (3). The effective two-photon wavefunction of type II SPDC is also different from that of the type I. The rectangular shaped biphoton [22] is shown graphically in Fig.13,

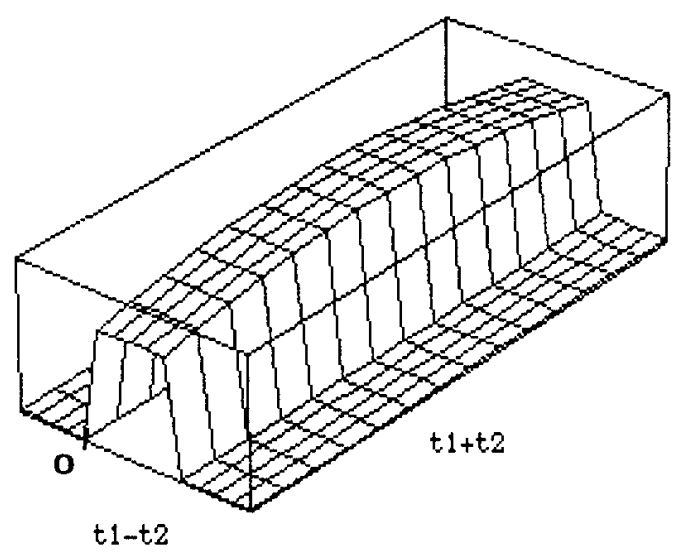

Figure 13: Schematic of a rectangular shaped type II biphoton wavepacket. 


$$
\Psi\left(t_{1}, t_{2}\right)=A_{0} \epsilon^{-\frac{-\tau_{p}^{2}\left(t_{1}+t_{2} 2^{2}\right.}{8}} \Pi\left(t_{1}-t_{2}\right) e^{-i \Omega_{s} t_{2}} e^{-i \Omega_{i} t_{2}}
$$

where $\Omega_{s}$ and $\Omega_{i}$ are the center frequency of the signal and the idler. respectirely, and

$$
\Pi\left(t_{1}-t_{2}\right)=\left\{\begin{array}{llc}
1 & \text { if } & 0 \leq t_{1}-t_{2} \leq D L \\
0 & \text { if } & \text { otherwise }
\end{array}\right.
$$

where $D \equiv 1 / u_{o}-1 / u_{e} \cdot u_{o}$ and $u_{e}$ are the group velocities of the o-ray and the $e$-ray inside the SPDC crystal. and $L$ is the length of the crystal.

\subsection{State Preparation and Quantum Teleportation}

The measurement of one subsystem of an entangled system composed of two subsystems may be thought of as a state preparation of the unmeasured subsystem. Quantum Teleportation can be looked at in this way. The nature of the prepared state may vary from being a pure state to being a random state depending on the measurement and on the initial entangled state. Te have recently studied the effects of measurements of finite duration on bipartite entangled states. One result of this work was the derivation of an integral equation that must be satisfied for accurate teleportation of arbitrary states. In addition. we have examined the conditions under which measurement of a single particle can lead to entanglement between one particle of an EPR pair and an independent particle. This latter work relates to attempts to prepare GHZ states from two independent down conversion pairs. We have derived the dependence of the prepared state on the measurement duration and spectral form of the entangled state. These results have been submitted for publication to Physical Review A.

\section{On Going Research}

\subsection{Three-Photon Entangled States}

Three-particle entangled state would reflect much richer physics of quantum entanglement. It will be shown in our future three-photon experiments with more suppressing and astonishment: howerer. providing much clear physics for quantum entanglement and for many fundamental concerns of quantum measurement theory.

One of our proposed investigations is to realize an entangled three-photon state, which is entangled in space-time or in spin. The state may be written in the simplified forms which is similar to the two-photon state of SPDC.

$$
\begin{array}{r}
|\Psi\rangle=\sum_{1.2 .3} \delta\left(\omega_{1}-\omega_{2}+\omega_{3}-C\right) \delta\left(\mathbf{k}_{1}+\mathbf{k}_{2}-\mathbf{k}_{3}-\mathbf{C}\right) \\
a_{1}^{\dagger}\left(\omega_{1}\left(\mathbf{k}_{1}\right)\right) a_{2}^{\dagger}\left(\omega_{2}\left(\mathbf{k}_{2}\right)\right) a_{3}^{\dagger}\left(\omega_{3}\left(\mathbf{k}_{3}\right)\right)|0\rangle
\end{array}
$$


where $C$ and $\mathrm{C}$ (vector) are two constants. which may correspond to the frequency and the wave vector of a laser pump.

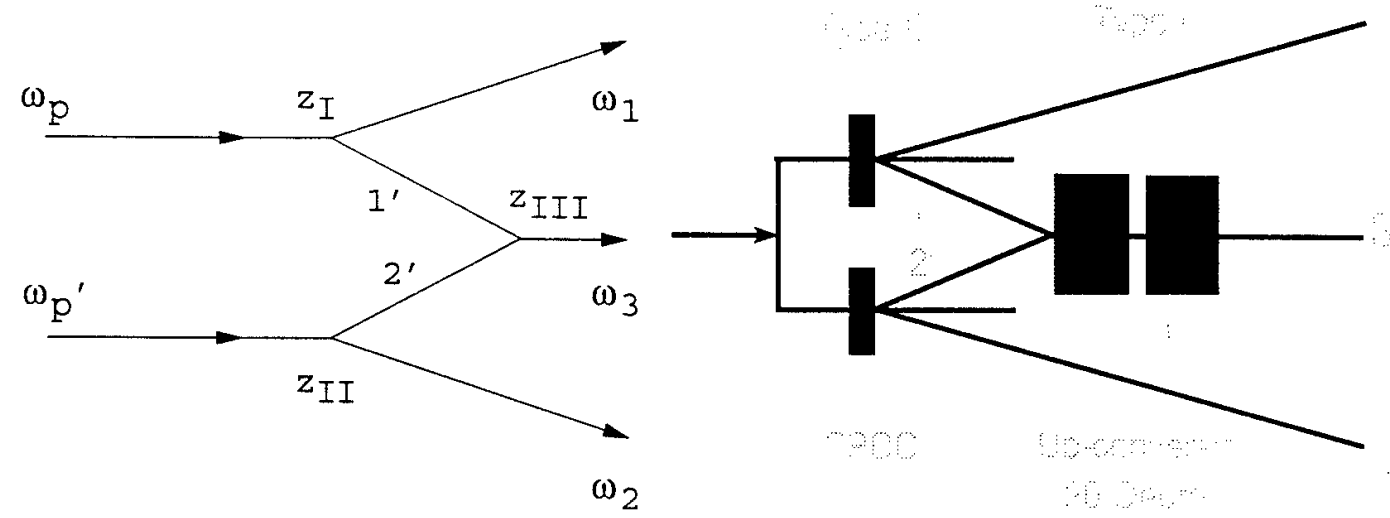

Figure 14: Principle Schematic of an experiment for triphoton generation. Two SPDC are pumped coherently to produce two signal-idler pairs. The idlers are upconverted in a SFG process to generate a third photon. The two signal photons along with the upconverted photon form a three-photon entangled state.

Theoretical study of triphoton generation has been published recently [23]. The idea is of using two coherently pumped SPDC to produce two pairs of signal-idler and "mixed up" the two idlers in a third Sum Frequency Generation (SFG) (also called "upconversion") to generate a third photon. The two signal photons along with the upconverted photon form a three-photon entangled state, or triphoton. The principle schematic is shown in Fig.14. Considering the pumps for the SPDCs are CW (coherent, for example. by splitting a laser beam into two) thus both $\omega_{p}$ and $\mathbf{k}_{p}$ can be considered as constants. It is easy to see that

$$
\omega_{1}+\omega_{2}+\omega_{3}=2 \omega_{p} . \quad \mathbf{k}_{1}+\mathbf{k}_{2}+\mathbf{k}_{3}=2 \mathbf{k}_{p}
$$

which implies the three-photon entangled state (11). However, the use of CW laser is not realistic in experiments because of the low efficiency of the SFG (single-photon level input). If a short pulsed pump laser is taken into account the analysis turns to be a bit complicated.

The following calculation applies for both CW and short pulse pumped SPDC-SFG processes.

These processes (SPDCs and SFG) result from the interaction of three electric fields 
inside a non-centrosymmetric crystal. The interaction Hamiltonian is given by [6].

$$
H(t)=\int_{1} d V \cdot \frac{2}{3} \epsilon_{0} \succ E_{3} E_{a} E_{b}
$$

where the integral is orer the volume of the crrstal and $\chi$ is the second order nonlinear electric susceptibility tensor. In SPDC a pump photon $\left(E_{3}=E_{p}\right)$ is annihilated and a pair of photons, called the signal and the idler $\left(E_{s}, E_{i}\right)$. are created. In SFG a pair of photons are annihilated to create a third photon. $E_{3}$. For the SPDC treated here, the electric field of the pump is taken to be classical, while the signal and idler fields are quantized. The pump beam is linearly polarized and propagates in the $z$-direction. It has a central frequency $\Omega_{p}$ and an envelope of arbitrary shape, $\tilde{E}_{p}$,

$$
\begin{aligned}
& E_{p}(z, t)=e^{-i \Omega_{p} t} \tilde{E}_{p}(z . t) \\
& \tilde{E}_{p}(z . t)=\int d \nu_{p} \bar{E}_{p}\left(\nu_{p}\right) e^{i k_{p}\left(\Omega_{p}+\nu_{p}\right) z-i \nu_{p} t}
\end{aligned}
$$

For simplifying the calculation. we confine ourselves to ignore the transverse coordinates. The positive frequency part of the signal field is defined by

$$
E_{s}^{(+)}=\sum_{\omega} \frac{e_{\omega}}{n_{s}(\omega)} a_{s}\left(k_{s}(\omega)\right) e^{i\left(k_{s}(\omega) \varepsilon-\omega t\right)}
$$

where $a(k)$ is the annihilation operator of a photon with wave number $k, e_{\omega}=\sqrt{\frac{\hbar \omega}{2 \epsilon_{0} l_{Q}}}$, $n(\omega)$ is the index of refraction of the crystal. and $V_{Q}$ is the quantization volume. For $\mathrm{SFG}$, all three fields are treated quantum mechanically.

The state vector of the triphoton is derived from third order perturbation:

$$
|\Psi\rangle=\left(-\frac{i}{\hbar}\right)^{3} \int_{\infty}^{\infty} d t_{I} \int_{-\infty}^{\infty} d t_{I I} \int_{-\infty}^{\infty} d t_{I I I} T\left[H\left(t_{I}\right) H\left(t_{I I}\right) H\left(t_{I I I}\right)\right]|0\rangle
$$

where $T$ is the time ordering operator and $|0\rangle$ is the racuum state. Keeping only the terms of interest.

$$
|\Psi\rangle=\sum_{k_{1}, k_{2}, k_{3}} F\left(k_{1}, k_{2}, k_{3}\right) a_{1}^{\dagger}\left(k_{1}\right) a_{2}^{\dagger}\left(k_{2}\right) a_{3}^{\dagger}\left(k_{3}\right)|0\rangle
$$

where the three-photon spectral function. $F$, is given by

$$
\begin{aligned}
F\left(k_{1}, k_{2}, k_{3}\right)= & g \int d \omega_{p} \int d \omega_{p}^{\prime} \bar{E}_{p}\left(\omega_{p}-\Omega_{p}\right) \bar{E}_{p}\left(\omega_{p}^{\prime}-\Omega_{p}\right) \\
& \delta\left(\omega_{p}+\omega_{p}^{\prime}-\omega_{1}-\dot{\omega}_{2}-\omega_{3}\right) U\left(\omega_{p}, \omega_{p}^{\prime}, \omega_{1}, \omega_{2}, \omega_{3}\right)
\end{aligned}
$$

and

$$
\begin{aligned}
U\left(\omega_{p} \cdot \dot{\omega}_{p}^{\prime}, \omega_{1}, \omega_{2}, \omega_{3}\right)= & \sum_{k_{1^{\prime}}, k_{2^{\prime}}} h\left(k_{p}-k_{1}-k_{1^{\prime}}\right) \times \\
& \times h\left(k_{p}^{\prime}-k_{2}-k_{2^{\prime}}\right) h\left(k_{1^{\prime}}-k_{2^{\prime}}-k_{3}\right) \\
& \times d\left(\omega_{p}-\omega_{1}-\omega_{1^{\prime}}\right) d\left(\omega_{p}^{\prime}-\omega_{2}-\omega_{2^{\prime}}\right) .
\end{aligned}
$$


The wave numbers are defined by

$$
k_{j}=\frac{\dot{u}_{j} n_{j}\left(\mathfrak{i}_{j}\right)}{c} .
$$

and

$$
d(x)=\frac{1}{x+i \epsilon}
$$

arises from the integration orer time at each vertex. The last time integral gives the Dirac delta function.

$$
h(x)=\int_{-L}^{0} e^{-i x z} d z
$$

is the integral over the crystal for the collinear case where we have assumed that the pump illuminates the entire crystal. Finally, $g$, is a function of all the slowly varying parameters and may be taken as constant.

The interaction will be a maximum when the phase matching conditions, $\omega_{p}=$ $\omega_{1}+\omega_{1^{\prime}}^{\prime} . \quad \omega_{p^{\prime}}=\omega_{2}+\omega_{2^{\prime}} . \quad \omega_{3}=\omega_{1^{\prime}}+\omega_{2^{\prime}}, \quad k_{p}=k_{1}+k_{1^{\prime}}, \quad k_{p^{\prime}}=k_{2}+k_{2^{\prime}}, \quad k_{3}=k_{1^{\prime}}+k_{2^{\prime}}$ are met.

Let $\omega_{r}=\Omega_{r}+\nu_{r}$ where we may assume that $\left|\nu_{r}\right|<<\Omega_{r}$. Expanding the wave vectors to first order we get

$$
k_{r}=K_{r}+\frac{\nu_{r}}{u_{r}\left(\Omega_{r}\right)}
$$

where $u_{r}\left(\Omega_{r}\right)$ is the group velocity of the r-beam. To ensure the interaction is coherent over the entire illuminated region. the crystal is cut so that for $r=1.2$

$$
\begin{aligned}
K_{r}+K_{r^{\prime}} & =K_{p} \\
K_{1}+K_{2} & =K_{3} \\
\Omega_{r}+\Omega_{r^{\prime}} & =\Omega_{p} \\
\Omega_{1}+\Omega_{2}+\Omega_{3} & =2 \Omega_{p}
\end{aligned}
$$

It may not be easy to see the entanglement nature at this point. Let us examine the three-photon warefunction, or triphoton, which is similar to the concept of biphoton for the two-photon entangled state.

In practice. the $\Omega_{1}, \Omega_{2}$, and $\Omega_{3}$ beams are not collinear and are separated by using pinhole apertures. The field at detector 1 can be written as

$$
E_{1}^{(+)}=\frac{1}{\sqrt{2}} \sum_{\omega} e_{\omega} a(k(\omega)) e^{-i \omega T_{1}}
$$

where $t_{1}=T_{1}-\frac{l_{1}}{c}$ and $l_{1}$ is the optical path length from the output face of the crystal to detector 1 . The fields at detector 2 and 3 are defined in a similar fashion. The probability amplitude of the triphoton is given by:

$$
A\left(T_{1}, T_{2}, T_{3}\right)=\left\langle 0\left|E_{1}^{(+)} E_{2}^{(+)} E_{3}^{(+)}\right| \Psi\right\rangle
$$


Evaluation of the triphoton probability amplitude vields:

$$
\begin{aligned}
A= & e^{-i\left(\Omega: T_{:}-\Omega_{2} T_{2}+\Omega_{3} T_{3}\right.} \cdot A_{0} \int_{-L}^{0} d z_{I I I} \Pi_{1}\left(T_{13}, z_{I I I}\right) \\
& \times \tilde{E}_{p}\left(0 . T_{3}+\frac{D_{31^{\prime}}}{D_{11^{\prime}}} D_{1 p} z_{I I I}+\frac{D_{p 1^{\prime}}}{D_{11^{\prime}}} T_{13}\right) \times \\
& \Pi_{2}\left(T_{23 .} \cdot z_{I I I}\right) \tilde{E}_{p}\left(0 . T_{3}+\frac{D_{32^{\prime}}}{D_{22^{\prime}}} D_{2 p} z_{I I I}+\frac{D_{p 2^{\prime}}}{D_{22^{\prime}}} T_{23}\right)
\end{aligned}
$$

where $T_{i 3}=T_{i}-T_{3}, D_{j k}=\frac{1}{u_{j}}-\frac{1}{u_{k}}$, and $\tilde{E}_{p}(0, t)$ is defined in Eq. (14). The arguments of the pump envelopes are the times of the SPDC. The ח's are defined by

$$
\Pi_{i}\left(T_{i 3}, z_{I I I}\right)=\begin{array}{lc}
1 & \left\{z_{I I} D_{3 i^{\prime}}>T_{i 3}-D_{i i^{\prime}} L\right\} \cap\left\{z_{I I I} D_{3 i}<T_{i 3}\right\} \\
0 & \text { otherwise }
\end{array}
$$

where $\mathrm{i}=1.2$ and $\cap$ is the intersection operator. The $\Pi_{i}$ function arises from the contour

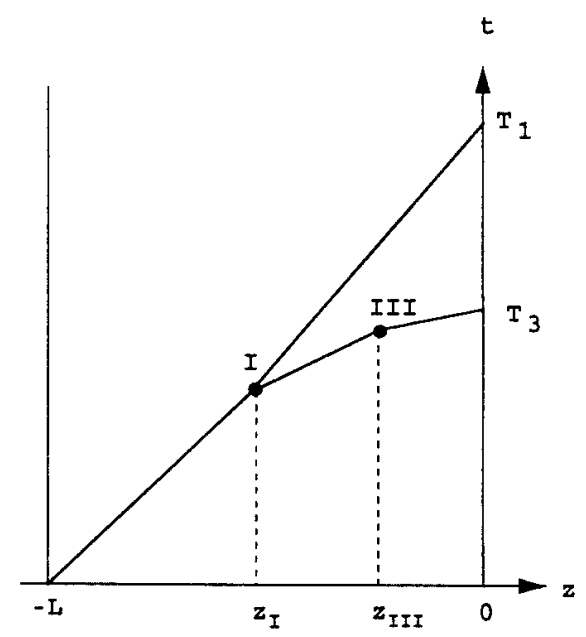

Figure 15: Interaction I precedes interaction II and the detection time difference between photons 1 and 3 is $T_{13}=-D_{11^{\prime} z_{I}}+D_{31^{\prime} z_{I I I}}$

integration over the first two interactions. $z_{I}$ and $z_{I I}$. Its form is determined by the time ordering of the events. Perhaps the easiest way to understand this is to explore the space-time relationship between the interactions. From Fig. 15 it is easy to see that

$$
T_{13}=-D_{11^{\prime} z_{I}}+D_{31^{\prime}} z_{I I I}
$$

where $-L \leq z_{I}<z_{I I I} \leq 0$. The maximum value for $z_{I I I}$ is 0 . The minimum value of $z_{I I I}$ must be larger than the minimum of $z_{I}$, so Eq. (24) leads to (23). 


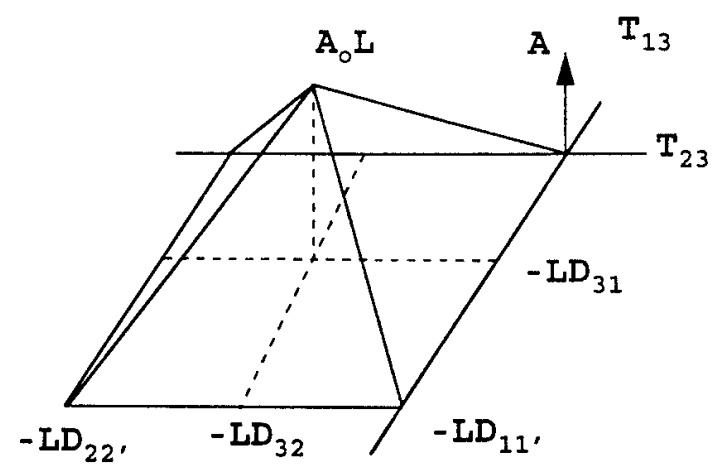

Figure 16: The CW triphoton wavepacket is pyramindal in shape.

For a monochromatic pump. $\tilde{E}_{p}\left(0 . \nu_{p}\right)=1$ and $|A|$ reduces to a function of $T_{13}$ and $T_{23}$

$$
|A|=\left|A_{0} \int_{-L}^{0} d z_{I I I} \Pi_{I}\left(T_{13 .} z_{I I I}\right) \Pi_{I I}\left(T_{23}, z_{I I I}\right)\right|
$$

Generally:

$$
|A|=A_{0} \times \min \left\{0, z_{u}-z_{l}\right\} \mid
$$

where $z_{u}$ and $z_{l}$ are determined by the overlap of the $\Pi$ functions. Evaluation of the integral requires specification of $D_{31}, D_{31^{\prime}}, D_{32}, D_{32^{\prime}}$. If $\left\{D_{31}, D_{32}\right\}>0$ and $\left\{D_{31^{\prime}}, D_{32^{\prime}}\right\}<$ 0 . Eq. (23) gives $z_{u}=0$ and $z_{l}=\max \left\{-L \cdot \frac{T_{13}}{D_{3}} \cdot \frac{T_{23}}{D_{32}}\right\}$. As shown in Fig.16. the triphoton probability amplitude is maximum when the $\Pi$ functions completely overlap. i.e.

$$
z_{i}=-L=\frac{T_{13}}{D_{31}}=\frac{T_{23}}{D_{32}} .
$$

and is zero when they do not, i.e. if $T_{13} / D_{13}$ or $T_{23} / D_{23}$ lies outside the interval [ $\left.-L .0\right]$. It follows that the triphoton assumes a pyramidal shape with the peak. $A_{0} L$.

The form of the triphoton for the pulse pump case is given by Eq. (22). For a Gaussian pump envelope, $\tilde{E}_{p}(0, t)=e^{-\left(\frac{t}{\sigma}\right)^{2}}$. the triphoton probability amplitude is given by:

$$
\left|A\left(T_{1}, T_{2}, T_{3}\right)\right|=U\left(\kappa_{2} \Upsilon_{1}-\kappa_{1} \Upsilon_{2}\right) V\left(\kappa_{1} \Upsilon_{1}+\kappa_{2} \Upsilon_{2}, z_{u}, z_{l}\right)
$$

where :

$$
\begin{aligned}
U(t) & =e^{-\left(\frac{t}{\sigma^{\prime}}\right)^{2}} \\
V(t) & =V_{0}\left(\operatorname{erf}\left(\frac{t}{\sigma \prime}+\frac{\sigma \prime}{\sigma^{2}} z_{u}\right)-\operatorname{erf}\left(\frac{t}{\sigma^{\prime}}+\frac{\sigma^{\prime}}{\sigma^{2}} z_{l}\right)\right) \\
\sigma^{\prime} & =\sigma \sqrt{\kappa_{1}^{2}-\kappa_{2}^{2}} \\
\Upsilon_{i} & =T_{3}+\frac{D_{p i^{\prime}}}{D_{i i^{\prime}}} T_{i 3}
\end{aligned}
$$




$$
\kappa_{i}=D_{i p} \frac{D_{3 i^{\prime}}}{D_{i i^{\prime}}}
$$

and $z_{u}\left(T_{13}, T_{23}\right)$ and $z_{1}\left(T_{13} . T_{23}\right)$ are defined as for the monochromatic pump case. It is difficult to visualize the three dimensional probability amplitude, $\left|A\left(T_{1}, T_{2}, T_{3}\right)\right|$ so let us examine the conditional probability amplitude $A\left(T_{1}, T_{2} . T_{3}=t\right) \mid$. This conditional probability amplitude specifies the probability amplitude distribution for photon 1 and photon 2 for a given detection time for photon 3. It is shown in Fig.17

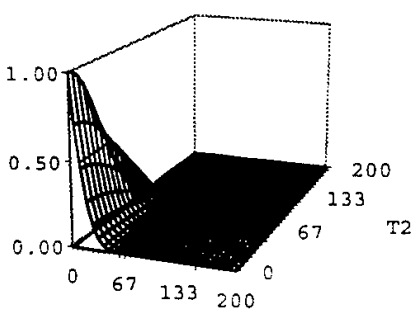

$\mathrm{T} 3=0 \mathrm{Es}$

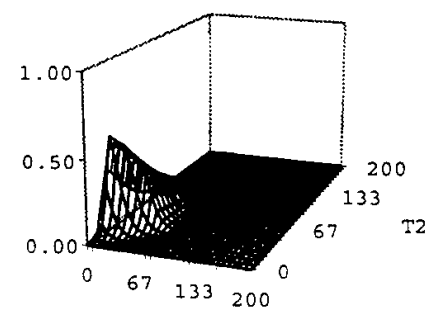

$\mathrm{T} 3=40 \mathrm{fs}$

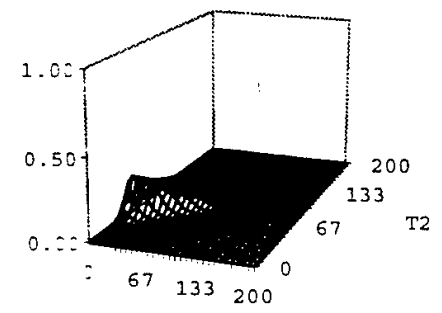

$\mathrm{T} 3=80 \mathrm{fS}$

Figure 17: The pulsed triphoton wavepacket at a given detection time of photon 3. which is a conditional probability amplitude distribution for photon 1 and photon 2 .

An interesting consequence of the narrow pulse pump case is the restriction on the sense of the $D$ 's. Take. for example. the case where the group velocity of one of the idlers (primed photons) is greater than the pump group relocity and the other idler's group relocity is less. It is clear that the triphoton probability is zero unless all three interactions occur simultaneously. The only practical realization of the pulse pump triphoton is when both idler photon's group velocities are greater (or less) than the pump group velocity: This condition is easy to be satisfied.

A three-photon entangled state has been calculated for both CW and pump pulse case. For experimental realization, only the pulse case will lead to reasonable counting rates. This limitation is due to the inherent low efficiencr of the upconversion. For a $100 \mathrm{fs}$ pulsed $\mathrm{CW}$ mode lock pump laser operating at $100 \mathrm{MHz}$, we may be able to achieve 1 pair per pulse for the SPDCs. Considering a $10^{-i}$ efficiency for the single photon SFG, we could achieve 10 coincidence counts per second. To increase the efficiency of single photon SFG is very important for the final realization of the triphoton. It has been our major experimental effort in the first year.

After the succesful generation of triphoton we shall be able to demonstrate a new set of exciting three-photon physics, including the space-time entanglement as well as the spin entanglement. 


\subsection{Quantum Teleportation with Complete Set Bell State Mea- surement}

The idea of quantum teleportation is to utilize the nonlocal correlations between an Einstein-Podolsky-Rosen pair of particles [3] to prepare a quantum system in some state, which is the exact replica of an arbitrary unknown state of a distant individual system [24]. Three experiments in this direction were published recently $[25,26,27]$.

The following conditions must be satisfied in any claim for quantum teleportation: (i) the input quantum state. which is teleported in the experiment must be an arbitrary state, (ii) there must be an output quantum state which is an "instantaneous copy" of the input quantum state. (iii) the Bell state measurement (BSM) must be able to distinguish the complete set of the orthogonal Bell states so that the input state can be teleported with certainty, and (iv) for any input state. the teleportation must be deterministic. not "statistical". Unfortunately, non of the above three published experiments satisfy" all of the requirements.

We have started to demonstrate a quantum teleportation scheme which satisfies all four of the above conditions. The input state is an arbitrary polarization state and the BSM can distinguish the complete set four orthogonal Bell states. Teleportation of a quantum state can thus occur with certainty in principle. This is because the BSM is based on nonlinear interactions which are necessary and non-trivial physical processes for quantum teleportation $[29,30]$. Another point we like to emphasize is that the Bell state measurement is deterministic in this scheme. This is important for the teleportation of a state. which contains real information.

The basic elements of the experiment are schematically shown in Fig. 18. Just as the original proposal of quantum teleportation [24], it consists of four essential parts: (a) the input state. (b) the EPR pair, (c) Alice (who performs the BSM of the input state and her EPR particle). and (d) Bob (who carries out unitary operations on his EPR particle). The input quantum state is an arbitrary polarization state given by,

$$
\left|\Psi_{1}\right\rangle=\alpha\left|0_{1}\right\rangle+3\left|1_{1}\right\rangle,
$$

where $|0\rangle$ and $|1\rangle$ represent the two orthogonal linear polarization bases (specifically in this paper) $|H\rangle$ (horizontal) and $|V\rangle$ (vertical) respectively: $\alpha$ and $\beta$ are two arbitrary complex amplitudes with respect to the $|0\rangle$ and $|1\rangle$ bases and satisfy the condition $|\alpha|^{2}+|\beta|^{2}=1$. The EPR pair shared by Alice and Bob is prepared by spontaneous parametric down contersion (SPDC) [31] as.

$$
\left\{\Psi_{23}\right\rangle=\frac{1}{\sqrt{2}}\left\{\left|0_{2} 0_{3}\right\rangle-\left|1_{2} 1_{3}\right\rangle\right\} .
$$

with the subscripts 2 and 3 as labeled in Fig.18. The complete state of the three particles before Alice's measurement is then.

$$
\left|\Psi_{123}\right\rangle=\frac{\alpha}{\sqrt{2}}\left\{\left|0_{1} 0_{2} 0_{3}\right\rangle-\left|0_{1} 1_{2} 1_{3}\right\rangle\right\}
$$




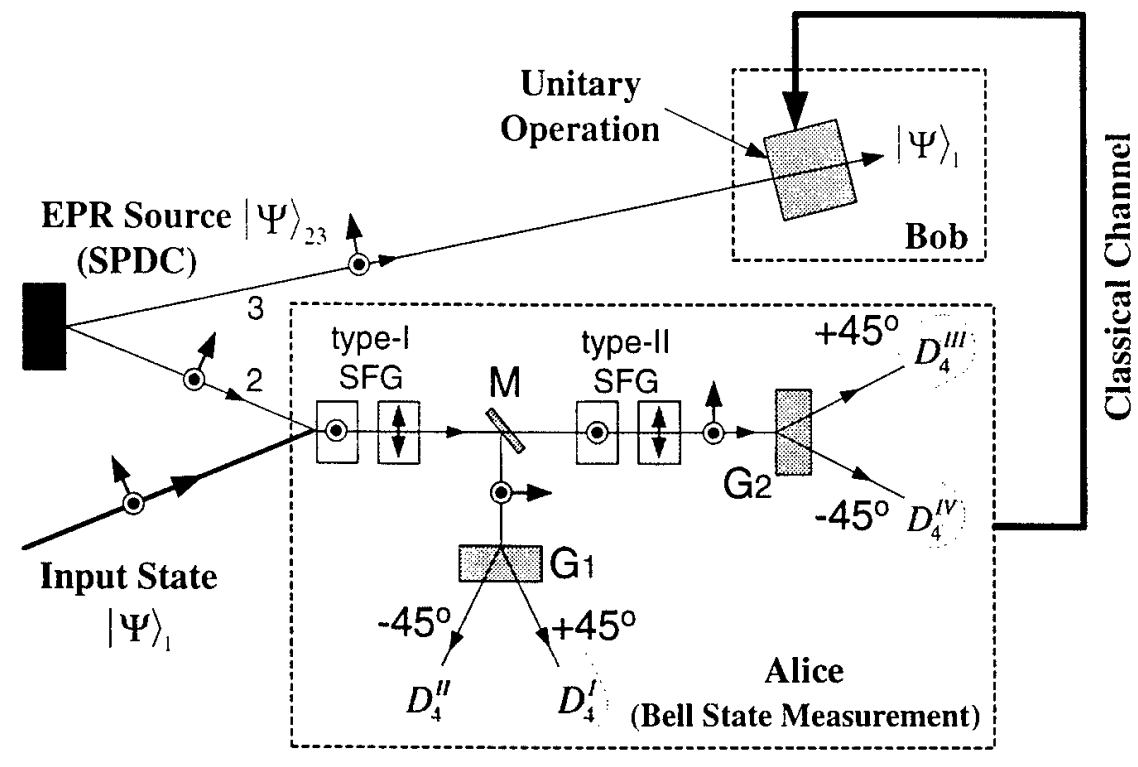

Figure 18: Schematic of quantum teleportation with complete BS.M. Nonlinear interactions (SFG) are used to perform the BS.M. $\odot$ and $\uparrow$ represent the respective horizontal and rertical orientations of the optic axes of the crystals.

$$
+\frac{3}{\sqrt{2}}\left\{\left|1_{1} 0_{2} 0_{3}\right\rangle-\left|1_{1} 1_{2} 1_{3}\right\rangle\right\}
$$

The four Bell states which form a complete orthonormal basis for particle 1 and particle 2 are usually represented as.

$$
\begin{aligned}
\left|\Phi_{12}^{( \pm)}\right\rangle & =\frac{1}{\sqrt{2}}\left\{\left|0_{1} 0_{2}\right\rangle \pm\left|1_{1} 1_{2}\right\rangle\right\} \\
\left|\Psi_{12}^{( \pm)}\right\rangle & =\frac{1}{\sqrt{2}}\left\{\left|0_{1} 1_{2}\right\rangle \pm\left|1_{1} 0_{2}\right\rangle\right\} .
\end{aligned}
$$

State (32) can now be re-written in the following form based on the above orthonormal Bell states,

$$
\begin{aligned}
& \left|\Psi_{123}\right\rangle=\frac{1}{2}\left\{\quad\left|\Phi_{12}^{(+)}\right\rangle\left(\alpha\left|0_{3}\right\rangle-\beta\left|1_{3}\right\rangle \quad\right)\right. \\
& +\left|\Phi_{12}^{(-)}\right\rangle\left(\alpha\left|0_{3}\right\rangle+\beta\left|1_{3}\right\rangle\right) \\
& \left.+\Psi_{12}^{(+)}\right\rangle\left(-\alpha\left|1_{3}\right\rangle+\beta\left|0_{3}\right\rangle\right) \\
& \left.+\left|\Psi_{12}^{(-)}\right\rangle\left(-\alpha\left|1_{3}\right\rangle-\beta\left|0_{3}\right\rangle\right) \quad\right\} \text {. }
\end{aligned}
$$

To teleport the state of particle 1 to particle 3 reliably. Alice must be able to distinguish her four Bell states by means of the BSM performed on particle 1 and her EPR share (particle 2). She then tells Bob through a classical channel to perform a 
corresponding linear unitary operation on his EPR share (particle 3) to obtain an exact replica of the state of particle 1 . This completes the process of quantum teleportation.

In our scheme. the Bell State Measurement is based on nonlinear interactions: optical Sum Frequency Generation (SFG) (or "upconversion"). Four SFG nonlinear crystals are used for "measuring" and "distinguishing" the complete set of the four Bell states. Photon 1 and photon 2 mar interact either in the two type-I crystals or in the two type-II crystals to generate a higher frequency photon (labeled as photon 4). The projection measurements on photon 4 (either the $45^{\circ}$ or $135^{\circ}$ direction) correspond to the four Bell states of photon 1 and photon 2. $\left|\Phi_{12}^{( \pm)}\right\rangle$and $\left|\Psi_{12}^{( \pm)}\right\rangle$.

The first type-I SFG crystal converts two $|V\rangle$ polarized photons $\left|1_{1} 1_{2}\right\rangle$ into a single horizontal polarized photon $\left|H_{4}\right\rangle$. Likewise, the second type-I SFG crystal converts two $|H\rangle$ polarized photons $\left|0_{1} 0_{2}\right\rangle$ into a single vertical polarized photon $\left|V_{4}\right\rangle$. The first and the last terms on the right-hand side in Eq.(32) thus become,

$$
\left|\Psi_{43}\right\rangle=\alpha\left|V_{4} 0_{3}\right\rangle-\beta\left|H_{4} 1_{3}\right\rangle .
$$

Dichroic beamsplitter $M$ reflects only SFG photons to the $45^{\circ}$ polarization projector $G_{1}$. Two detectors $D_{4}^{I}$ and $D_{4}^{I I}$ are placed at the $45^{\circ}$ and $135^{\circ}$ output ports of $G_{1}$ respectively. Denoting the $45^{\circ}$ and $135^{\circ}$ polarization bases by $\left|45^{\circ}\right\rangle$ and $\left|135^{\circ}\right\rangle$, the state $\left.\Psi_{43}\right\rangle$ can be re-written as.

$$
\begin{aligned}
\left|\Psi_{43}\right\rangle= & \frac{1}{\sqrt{2}}\left\{\left|45^{\circ}\right\rangle_{4}\left(a\left|0_{3}\right\rangle-\beta\left|1_{3}\right\rangle\right)\right. \\
& \left.+\left|135^{\circ}\right\rangle_{4}\left(\alpha\left|0_{3}\right\rangle+\beta\left|1_{3}\right\rangle\right)\right\}
\end{aligned}
$$

which gives.

$$
\begin{aligned}
|\Psi\rangle_{3 \mid D_{1}^{I}} & =\alpha\left|0_{3}\right\rangle-\beta\left|1_{3}\right\rangle \\
|\Psi\rangle_{3 \mid D_{4}^{I I}} & =\alpha\left|0_{3}\right\rangle+\beta\left|1_{3}\right\rangle
\end{aligned}
$$

i.e.. if detector $D_{4}^{I}\left(45^{\circ}\right)$ is triggered, the quantum state of photon 3 (on Bob side) is: $\left|\Psi_{3}\right\rangle=\alpha\left|0_{3}\right\rangle-\beta\left|1_{3}\right\rangle$ and if detector $D_{4}^{I I}\left(135^{\circ}\right)$ is triggered, the quantum state of photon 3 is: $\left|\Psi_{3}\right\rangle=\alpha\left|0_{3}\right\rangle+\beta\left|1_{3}\right\rangle$.

As we have analyzed above. the $45^{\circ}$ and the $135^{\circ}$ polarized type-I SFG components in Eq. (34) correspond to the superposition of $\left|0_{1} 0_{2}\right\rangle$ and $\left|1_{1} 1_{2}\right\rangle$ which are the respective Bell states $\left|\Phi_{12}^{(+)}\right\rangle$and $\left|\Phi_{12}^{(-)}\right\rangle$.

Similarly, the other two Bell states are distinguished by the type-II SFG's. The states $\left.0_{1} 1_{2}\right\rangle$ and $\left|1_{1} 0_{2}\right\rangle$ are made to interact in the first and the second type-II SFG crystals respectively to generate a higher frequency photon with either horizontal (the first type-II SFG) or vertical (the second type-II SFG) polarization. A $45^{\circ}$ polarization projector $G_{2}$ is used after the type-II SFG crystals and two detectors $D_{4}^{I I I}$ and $D_{4}^{I V}$ are placed at the $45^{\circ}$ and the $135^{\circ}$ output ports of $G_{2}$ respectively. On the new bases 
of $45^{\circ}$ and $135^{\circ}$ for the SFG photon. the second and the third terms on the right-hand side in Eq.(32) thus become.

$$
\begin{aligned}
\left|\Psi_{43}\right\rangle= & \left.\left.\frac{1}{\sqrt{2}}\left\{45^{\circ}\right\rangle_{4}\left(-\alpha 1_{3}\right\rangle+3: 0_{3}\right\rangle\right) \\
& \left.\left.\left.+135^{\circ}\right\rangle_{4}\left(-\alpha \cdot 1_{3}\right\rangle-\beta\left|0_{3}\right\rangle\right)\right\}
\end{aligned}
$$

which gives,

$$
\begin{aligned}
& |\Psi\rangle_{3 \mid D_{4}^{I I I}}=-\alpha\left|1_{3}\right\rangle+\beta\left|0_{3}\right\rangle, \\
& |\Psi\rangle_{3 \mid D_{4}^{I I}}=-\alpha\left|1_{3}\right\rangle-\beta\left|0_{3}\right\rangle,
\end{aligned}
$$

i.e., if detector $D_{4}^{I I}\left(45^{\circ}\right)$ is triggered, the quantum state of photon 3 is: $\left|\Psi_{3}\right\rangle=$ $-\alpha\left|1_{3}\right\rangle+\beta\left|0_{3}\right\rangle$ and if detector $D_{4}^{I V}\left(135^{\circ}\right)$ is triggered. the quantum state of photon 3 is: $\left|\Psi_{3}\right\rangle=-\alpha\left|1_{3}\right\rangle-3\left|0_{3}\right\rangle$.

The $45^{\circ}$ and the $135^{\circ}$ polarized type-II SFG components correspond to the superposition of $\left|0_{1} 1_{2}\right\rangle$ and $\left|1_{1} 0_{2}\right\rangle$ which are the Bell states $\left|\Psi_{12}^{(+)}\right\rangle$and $\left|\Psi_{12}^{(-)}\right\rangle$respectively.

To obtain the exact replica of the state of Eq.(30). Bob needs simply to perform a corresponding unitary transformation after learning from Alice which of her four detectors. $D_{4}^{I}, D_{4}^{I I} . D_{4}^{I I I}$. or $D_{4}^{I V}$, is triggered.

\section{Publications}

\section{(1) Publications in Refereed Journals}

- "A Delayed Quantum Eraser". Phys. Rer. Lett.. Vol. 60, 1 (2000) (Y.H. Kim, R. Yu. S.P. Kulic. Y.H. Shih. and M.O. Scully).

- "Entanglement and State Preparation". Phys. Rev. A. Vol. 61. 022311 (2000) (M. H. Rubin).

- "Separability and Fourier Representations of Density Matrices", Phys. Rev. A, Accepted for publication, (2000) (A.O. Pittenger and M.H. Rubin).

- "Vote on Separability of the Werner States in Arbitrary Dimensions". Book Chapter, Special Issue of Optics Comm.. (2000) (A.O. Pittenger and M.H. Rubin).

- "Quantum Entanglement: from Popper's Experiment to Quantum Eraser", Book Chapter, Special Issue of Optics Comm.. (2000) (Y.H. Shih and Y.H. Kim).

- "Popper's Experiment: Uncertainty and Entanglement". Foundations of Physics, Vol. (1999) (T.H. Kim and Y.H. Shih). 
- "Quantum interference by two temporally distinguishable pulses". Phys. Rev. A. Rapid Comm.. Yol. 60. R37 (1999) (Y.H. Kim, M.Y. Chekhova, S.P. Kulik, and Y.H. Shih .

- "Experimental Study of $\$ Subsystem in an Entangled Two-photon State", Phys. Rev. A, Vol. 60. 2685 (1999) (D.V. Strekalov. Y.H. Kim. and Y.H. Shih).

- "Optical Wigner Functions for Two-photon Entangled Wave Packets", JOSB, Vol. 16, 1730 (1999) (Y. Ben-Aryeh. Y.H. Shih. and M.H. Rubin).

(2) Conference Publications (* Invited Paper)

- *New Quantum Eraser Experiments", 30th Winter Colloquium on the Physics of Quantum Electronics". Snowbird, Utah, (2000) (Y.H. Shih).

- * "Quantum Eraser and Causality". Symposium on Quantum Coherence and Entanglement. Taxes At and M University, (2000) (Y.H. Shih).

- * "From Popper's Experiment to Quantum Eraser". International Conference on Laser Physics and Quantum Optics. Shanghai. China. (1999) (Y.H. Shih).

- * "From Popper s Experiment to Quantum Eraser". Workshop on Fundamental Problems in Quantum Theory: UMBC. (1999) (Y.H. Shih).

- * "Entanglement and State Preparation", Workshop on Fundamental Problems in Quantum Theory: LIIBC. (1999) (Rubin).

- * "Two-Photon Physics: from Popper's Experiment to 'quantum Eraser", Sixth International Conference on Squeezed States and Lncertainty Relations. Naples. Italy, (1999) (Y.H. Shih).

- * "Entanglement and State Preparation". Sixth International Conference on Squeezed States and Lncertainty Relations, Naples, Italy. (1999) (Rubin).

- "A Quantum Eraser Experiment", Technical Digest of QELS'99, Baltimore, (1999) (Y.H. Kim and Y.H. Shih).

- "Two-photon Interference between Two Well-Separated Pulses". Technical Digest of QELS'99. Baltimore. (1999) (Y.H. Kim, M.V. Chekhora. S.P. Kulik, and Y.H. Shih).

- "Experimental Realization of Popper's Experiment", Technical Digest of QELS'99, Baltimore, (1999) (Y.H. Kim. and Y.H. Shih).

- * "Entanglement and State Preparation", Quantum Interferometry III, Trieste, Italy, (1999) (Rubin). 
- * "From Popper's Experiment to 'Quantum Eraser" - Are we talking the same photon?" Quantum Interferometry III. Trieste, Italy. (1999) (Y.H. Shih).

- * "Two-Photon Physics: from Popper's Experiment to "quantum Eraser". 29th Winter Colloquium on the Physics of Quantum Electronics. Snowbird. Ltah, (1999) (Y.H. Shih).

- * "Two-Photon Physics: from 'Ghost" Image to 'quantum Eraser"', Symposium on Novel Optical Materials. Texas A and II University; Texas. (1999) (Y.H. Shih).

\section{References}

[1] Y.H. Kim and Y.H. Shih. Foundations of Physics, 29, 1849 (1999).

[2] Y.H. Kim, R. Yu, S.P. Kulik. Y.H. Shih. and M.O. Scully. Phys. Rev. Lett., 84. 1 (2000).

[3] A. Einstein. B. Podolsky and X. Rosen. Phys. Rer. 47. Tit (1935).

[4] K.R. Popper, Zur Kritik der Ungenauigkeisrelationen. Die Naturwissenschaften, 22. Helft, 48, 807 (1934): K. R. Popper. From the Postscript to the Logic of Scientific Discovery. edited br. E.I. Bitsakis and X. Tambakis, Gutenberg Publishing, (1984); K. Popper, Quantum Theory And The Schism In Physics, edited by W.W. Bartly, Hutchinson, London, 28 (1983). Amongst the most notable opponents to the "Copenhagen School" were Einstein-Podolsky-Rosen. de Broglie. Landé. and Karl Popper. One may not agree with Popper's philosophy (EPR classical reality as well) but once again. Popper's thought experiment gives yet another way of understanding the foundations of quantum theory.

[0] The use of a "point source" in the original Popper's proposal has been criticized. The basic argument is that a point source can never produce a pair of entangled particles which preserves two-particle momentum conservation. Howerer, a "point source" is not a necessary requirement for Popper's experiment. What we need is to learn the precise knowledge of a particle's position through quantum entanglement. Our experiment using a different approach.

[6] A. Yariv, Quantum Electronics. John Wiley and Sons. New York. (1989).

[i] D.N. Klyshko. Photon and Nonlinear Optics, Gordon and Breach Science. New York. (1988).

[8] T.B. Pittman. Y.H. Shih. D.V. Strekalov, and A.V. Sergienke. Phys. Rev. A. 52. R3429 (1995).

[9] M.O. Scully and K. Drühl. Phys. Rev. A 25, 2208 (1982). 
[10] ‥ Bohr, Naturwissenschaften. 16. 24j (1928).

[11] R. Feynman. R. Leighton. and M. Sands. The Feynman Lectures on Physics. Vol. III. Addison Wesley: Reading (1965).

[12] See Wheeler's "delayed choice". in Quantum Theory and Measurement, edited by J.A. Wheeler and W.H. Zurek. Princeton Lniv. Press (1983).

[13] M.H. Rubin, D.K. Klyshko, and Y.H. Shih. Phrs. Rev. A 50, 5122 (1994).

[14] D. Voss, Science. Vol. 274,527 (1996). This Nota Bene article calls attention to one of our experiment published in Phys. Rev. Lett.. 77, 1917 (1996). The title of this Nota Bene article "One plus one is not two" sends an important message: the physics of the entangled two-particle system is inherently different from that of the two individual particles.

[15] D.I. Strekalov, A.V. Sergienko. D.K. Klyshko. and Y.H. Shih, Phys. Rer. Lett., 74. 3600 (1995). T.B. Pittman. D.V. Strekalor. A.V. Sergienko, and Y.H. Shih, Rapid Comm., Phys. Rev. A. 52. 3429 (1995).

[16] D. Bohm. Quantum Theory. Prentice Hall. Englewood Cliffs, NJ. 1951.

[17] C.S. Wu and I. Shaknov. Phys. Rer. 77. 136 (1950).

[18] For a review, see J.F. Clauser and A.Shimony, Rep. Prog. Phys. 41, 1881 (1976); A.Aspect. P.Grangier, and G. Roger. Phys. Rer. Lett., 47, 460 (1981); A.Aspect, P.Grangier. and G. Roger. Phys. Rer. Lett.. 49. 91 (1982); A.Aspect, J. Dalibard. and G. Roger, Phys. Rev. Lett.. 49. 1804 (1982): Y.H. Shih and C.O. Alley, Phys. Rer. Lett. 61, 2921 (1988): Z.Y. Ou. and L. Mandel. Phys. Rev. Lett. 61. 54 (1988): J.G. Rarity and P.R. Tapster. Phys. Rev. Lett. 64, 2495 (1990): P.G. Kwiat, A.M. Steinberg, and R.Y. Chiao. Phys. Rev. A 47, 2472 (1993): T.E. Kiess, Y.H.Shih. A.V. Serienko. and C.O. Aller: Phys. Rev. Lett. 71, 3893 (1993); T.B. Pittman, Y.H. Shih, A.V. Sergienko. and M.H. Rubin, Phys. Rev. A. Vol. 51, 3495 (1995).

[19] E.Schrodinger, Naturwissenschaften 23.807.823.844(1935); a translation of these papers appears in Quantum Theory and Measurement, edited by J.A. Wheeler and II.H. Zurek, Princeton University Press. New York (1983).

[20] For example. Y.H. Shih. A.V. Sergienko, M.H. Rubin, T.E. Kiess, and C.O. Alley, Phrs. Rer. A 50. 23 (1994): Y.H. Shih. A.V. Sergienko. Phys. Lett. A. Vol. 186, 29 (1994).

[21] T.B. Pittman, Y.H. Shih. A.V. Sergienko. and M.H. Rubin, Phys. Rev. A. Vol. 51 , 3495 (1995). 
[22] A.Y. Sergienko and Y.H. Shih. J. of Opt. Soc. of America. B, Vol 12. 859 (1995).

[23] T.E. Keller. M.H. Rubin. and Y.H. Shih, Phys. Rer. A. 57. 2076 (1998).

[24] C.H. Bennett et al.. Phrss. Rev. Lett. 70. 1895 (1993).

[25] D. Bouwmeester et al. Nature 390. 575 (1997).

[26] D. Boschi et al.. Phys. Rev. Lett. 80, 1121 (1998).

[27] A. Furusawa et al.. Science 282, 706 (1998).

[28] Y.H. Kim, S.P. Kulik, and Y.H. Shih, to be published. Phys. Rev. Lett., (2000).

[29] Complete BSM cannot be performed with only linear elements. See, L. Vaidman and N. Yoran. Phys. Rev. A 59, 116 (1999); N. Lütkenhaus, J. Calsamiglia, and K.-A. Suominen. ibid. 59. 3295 (1999).

[30] D.N. Klyshko. JETP 87. 639 (1998).

[31] SPDC (Spontaneous Parametric Down Conversion) is an optical nonlinear interaction process in which a pair of entangled photons is generated when a laser beam (pump) is passing through a nonlinear crystal. The first experimental demonstration of its entanglement nature of SPDC by means of the nonlocal EPR correlation measurement of the EPR-Bohm-Bell state was peported in 1986: C.O. Alley and Y.H. Shih. Proceedings of the Second International Srmposium on Foundations of Quantum Mechanics in the Light of New Technology: ed. M.Namiki. (1986). This work was finally published in Physical Review Letters after two years refereeing: Y.H. Shih and C.O. Alley. Phys. Rev. Lett.. 61, 2921. (1988). To review the experimental and theoretical studies of quantum entanglement and SPDC. see Y.H. Shih. "Two-Photon Entanglement and Quantum Reality", Advances in Atomic, Molecular, and Optical Physics, ed., B. Bederson and H. Walther, Academic Press. Cambridge, $199 \bar{\top}$. 